\title{
Qualifikationsnachfrage und Arbeitsmarkteintritt in der Schweiz
}

\author{
Arbeit im erlernten Beruf, Berufswechsel oder \\ Arbeitslosigkeit
}

\author{
Helen Buchs • Barbara Müller • Marlis Buchmann
}

(C) Springer Fachmedien Wiesbaden 2015

Zusammenfassung: Berufsorientierte Bildungssysteme sind auf die Vermittlung von spezialisierten beruflichen Qualifikationen ausgelegt. Werden diese nach Abschluss einer beruflichen Grundbildung auf dem Arbeitsmarkt wenig nachgefragt, riskieren die Absolventen, die erworbenen berufsspezifischen Qualifikationen nicht verwerten zu können. Dies trifft nicht nur zu, wenn Arbeitsmarkteinsteiger arbeitslos werden, sondern auch, wenn sie den Beruf wechseln müssen. Für die drei verschiedenen Optionen des Arbeitsmarkteinstiegs - Arbeit im erlernten Beruf, Berufswechsel und Arbeitslosigkeit - wird erstens untersucht, welche Bedeutung das individuell passende und das fachfremde Stellenangebot hat. Zweitens wird analysiert, wie eine unterschiedlich ausgeprägte Nachfrage nach fachspezifischen Qualifikationen die Bedeutung ausbildungsbezogener und individueller Merkmale für den Berufseinstieg verändert. Die Schweiz mit ihrem berufsorientierten Bildungssystem und berufsfachlich segmentieren Arbeitsmarkt dient als empirische Referenz. Die multinominalen logistischen Regressionsmodelle basieren auf den Daten des Schweizer Jugendpanels PISA2000/TREE. Die Individualdaten von Lehrabgänger werden auf

\footnotetext{
Aus Gründen der besseren Lesbarkeit wird auf die gleichzeitige Verwendung männlicher und weiblicher Sprachformen verzichtet. Sämtliche Personenbezeichnungen gelten gleichwohl für beiderlei Geschlecht.
}

\footnotetext{
H. Buchs $(\bowtie) \cdot$ M. Buchmann

Soziologisches Institut, Universität Zürich,

Andreasstrasse 15, 8050 Zürich, Schweiz

E-Mail: buchs@soziologie.uzh.ch

M. Buchmann

E-Mail: buchmann@soziologie.uzh.ch

B. Müller

TREE, Universität Bern,

Fabrikstrasse 8, 3012 Bern, Schweiz

E-Mail: barbara.mueller@soz.unibe.ch
} 
Berufsebene, zeitpunkt- und regionsspezifisch mit dem individuell passenden und fachfremden Stellenangebot verknüpft, das auf den Stelleninseratendaten des Stellenmarktmonitors Schweiz (SMM) beruht. Dank dieser Verknüpfung von Angebot und Nachfrage nach beruflichen Qualifikationen auf der Mikroebene kann zum ersten Mal die grundlegende Bedeutung der Personalnachfrage für den Berufseinstieg nachgewiesen werden.

Schlüsselwörter: Stellenanzeigen · Qualifikationsnachfrage · Berufswechsel · Berufsausbildung $\cdot$ Arbeitsmarkteintritt

\section{Skill-demand and labor market entry in Switzerland}

Work in the certified occupation, occupational change or unemployment

Abstract: Vocational education systems (VET) convey occupation-specific skills to their students. If the demand for the particular skills is scarce at the time when VET graduates attempt to enter the labor market, they run the risk of not being able to make use of them. This is not only the case when VET graduates become unemployed but also when they have to change the occupation. This paper investigates, first, whether the vacancies in the occupation for which the VET graduate is trained and those for which he or she is not trained affect the likelihood of finding a job in the certified occupation, changing the occupation or becoming unemployed. Second, the paper examines how the strength of the demand for occupation-specific skills modifies the influence of educational and individual factors on the three options of labor market entry. Switzerland known for its vocational education system and occupation-specific segmented labor market serves as empirical reference. The multinomial logistic regression models are based on the Swiss PISA/TREE panel data, focusing on VET graduates. We link the individual data on VET graduates to the job advertisement data of the Swiss Job Market Monitor (SJMM), matching the occupation-specific vacancies and those outside the certified occupation by occupation, year, and region. The link between the supply and demand sides of occupational skills at the individual level of labor market entrants offers the unique opportunity to assess the fundamental significance of job opportunities for labor market entry.

Keywords: Vacancies $\cdot$ Skill demand $\cdot$ Occupational change $\cdot$ Vocational education · Labor market entry

\section{Einleitung}

Der Eintritt ins Erwerbsleben ist ein entscheidender und zugleich schwieriger Schritt im Lebenslauf junger Erwachsener. So hängt die längerfristige Positionierung auf dem Arbeitsmarkt zu einem guten Teil von einem geglückten Übergang in die Erwerbsarbeit ab (z. B. Bukodi und Dex 2010; Margolis et al. 2004; Pollmann-Schult und Büchel 2002; Scherer 2004; Vandenberghe 2010). 
Ist das Bildungssystem stark beruflich ausgerichtet und der Arbeitsmarkt entsprechend fachlich segmentiert, kommt der fachlichen Übereinstimmung zwischen der beruflichen Grundbildung und der beim Arbeitsmarkteintritt ausgeübten Tätigkeit eine große Bedeutung zu (Solga und Konietzka 1999). Ein etwaiger Berufswechsel droht die erworbenen fachspezifischen Qualifikationen längerfristig zu entwerten, da die berufliche Mobilität im Erwerbsverlauf schnell abnimmt (Büchtemann et al. 1993; Gangl 2003b; Sheldon 2005). Ein geringes Angebot an berufsspezifischen Stellen nach Abschluss der Ausbildung erhöht den Druck auf Absolventen beruflicher Grundbildungen einen Berufswechsel in Kauf zu nehmen, wollen sie einer Phase von Arbeitslosigkeit mit möglichen ,scarring“-Effekten (Gangl 2004; Helbling und Sacchi 2014; Vandenberghe 2010) entgehen.

Vor diesem Hintergrund untersucht der vorliegende Beitrag am Beispiel der Schweiz erstmals für einen segmentierten Arbeitsmarkt, unter welchen Bedingungen Absolventen beruflicher Grundausbildungen eine fachlich adäquate Beschäftigung finden, einen Berufswechsel vollziehen oder Arbeitslosigkeit in Kauf nehmen müssen. Dabei richtet sich das Augenmerk auf die in der Berufseinstiegsforschung stark vernachlässigten nachfrageseitigen Kräfte im Arbeitsmarkt. Bisher wurde die Rolle der Qualifikationsnachfrage für den Berufseinstieg nie auf der Mikroebene untersucht, weil dazu die entsprechenden Daten fehlten. Gerade in beruflich segmentierten Arbeitsmärkten sind jedoch allgemeine, auf der Makroebene angesiedelte Indikatoren für die Arbeitskräftenachfrage wenig aussagekräftig, da die Nachfrage nach Arbeitskräften in den einzelnen Segmenten stark variieren kann. Mit den Daten des Stellenmarktmonitors Schweiz (www.stellenmarktmonitor.ch) lassen sich erstmals personenbezogene Indikatoren für die Beschäftigungsmöglichkeiten erstellen, die das mit Bezug auf die erworbenen Qualifikationen individuell passende und das fachfremde Stellenangebot abbilden. Wir untersuchen, inwiefern das Volumen an passenden sowie fachfremden offenen Stellen die Wahrscheinlichkeit einer fachlich adäquaten Beschäftigung, eines Berufswechsel oder der Arbeitslosigkeit nach Abschluss einer beruflichen Grundbildung beeinflusst. Weiter zeigen wir auf, wie ausbildungsbezogene und individuelle Merkmale bei unterschiedlich starker passender Personalnachfrage den Berufseinstieg mitbestimmen.

Eine fachspezifische und personenbezogene Analyse der Personalnachfrage kann das Verständnis des Eintritts in einen fachlich segmentierten Arbeitsmarkt entscheidend verbessern. Denn erst das Zusammenspiel von angebots- und nachfrageseitigen Faktoren ergibt ein getreues Bild des Berufseinstiegs. Die Beschäftigungsmöglichkeiten zum Zeitpunkt des Ausbildungsabschlusses entscheiden über die Verwertung von erworbenen Qualifikationen und individuellen Ressourcen. Analysen unter Einbezug des Stellenangebots können so aufzeigen, ob und wie Beschäftigungsmöglichkeiten die Rolle der bisher hauptsächlich untersuchten ausbildungsbezogenen und individuellen Faktoren verändert. Die Ergebnisse für die Schweiz dürften für all diejenigen Länder von hohem Interesse sein, die sich ebenfalls durch berufsorientierte Bildungssysteme und einen entsprechend segmentierten Arbeitsmarkt auszeichnen (Deutschland, Österreich, Dänemark und Holland; siehe Schellenbauer et al. 2010; Wolter und Ryan 2011).

Der vorliegende Beitrag gliedert sich wie folgt: Zuerst werden die theoretischen Überlegungen zum Einfluss der Qualifikationsnachfrage und von ausbildungs- 
bezogenen und individuellen Faktoren auf die fachliche Adäquanz der Beschäftigung beim Arbeitsmarkteintritt dargelegt. Anschließend werden die verwendeten Daten und Methoden vorgestellt. Der Präsentation der Schätzresultate folgen einige Schlussfolgerungen.

\section{Stellenangebot und Berufseinstieg: Theoretische Überlegungen und Hypothesen}

Die institutionelle Ausgestaltung des Bildungssystems strukturiert den Arbeitsmarkteintritt wesentlich (Allmendinger 1989; Gangl 2003a). Es soll deshalb zuerst auf die Eigenheiten des berufsorientierten Bildungssystems am Beispiel der Schweiz eingegangen werden. Danach wenden wir uns den theoretischen Überlegungen zur Rolle des Stellenangebots und von ausbildungsbezogenen und individuellen Faktoren für die fachliche Adäquanz der Beschäftigung beim Arbeitsmarkteintritt zu.

\subsection{Berufsorientierte Bildungssysteme und fachlich segmentierte Arbeitsmärkte}

Berufsorientierte Bildungssysteme mit einer starken beruflichen Differenzierung der Ausbildungsgänge gehen mit einer berufsfachlichen Segmentierung des Arbeitsmarkts einher. Die berufsspezifischen Ausbildungszertifikate steuern den Zugang zu den fachlichen Teilarbeitsmärkten (Blossfeld und Mayer 1988; Sengenberger 1978). Die hohe Standardisierung der Ausbildungen verstärkt die enge Anbindung der beruflichen Möglichkeiten an die fachspezifischen Zertifikate (Blossfeld und Mayer 1988). Die beruflichen Ausbildungen unterscheiden sich weiter bezüglich der vermittelten Qualifikationsausstattung, bezüglich Umfang und Zusammensetzung der Qualifikationen. Auch diese prägt die Zugänge zum Arbeitsmarkt sowie Sozialstatus und Karrierechancen (Konietzka 2010, 2011). Insgesamt liefern die berufsspezifischen Ausbildungszertifikate genaue Informationen über die in der Ausbildung erworbenen Qualifikationen und sind deshalb die wichtigste Einstellungsvoraussetzung auf dem Arbeitsmarkt (Kriesi et al. 2010). Insbesondere zu Beginn der Erwerbskarriere ist die berufliche Grundbildung Hauptressource der Qualifikationen und entscheidend für die berufliche Positionierung (Witte und Kalleberg 1995).

Die Schweiz kann als Musterland für ein berufsorientiertes Bildungssystem mit einer hohen fachlichen Differenzierung und Standardisierung betrachtet werden. Mit rund zwei Drittel einer Alterskohorte absolviert weltweit der höchste Anteil Jugendlicher eine berufliche Grundbildung (Schellenbauer et al. 2010; Wolter und Ryan 2011). Die große Mehrheit der beruflichen Ausbildungen findet als sogenannte „duale" Berufsbildung statt. Beruflicher und allgemeinbildender Unterricht in einer Berufsschule ergänzt den Erwerb von berufspraktischen Kenntnissen und Fertigkeiten in einem Lehrbetrieb. Duale Berufslehren vermitteln somit nicht nur berufliche Qualifikationen, sondern garantieren auch betriebliche Sozialisation und Praxiserfahrung (Corsten und Lempert 1997). Der Schwerpunkt liegt bei dieser Ausbildungsform mehrheitlich beim Erwerb spezialisierter beruflicher Qualifikationen. Alternativ zur dualen Ausbildung in einem Betrieb können Berufslehren auch in einem vollschulisch organisierten Angebot absolviert werden. Bei dieser Ausbildungsform 
fehlt der berufspraktische und betriebliche Bezug. Schulische Ausbildungsangebote sind in Lehrgängen verbreitet, die verstärkt allgemeine Qualifikationen vermitteln. Gleichzeitig zur oder im Anschluss an die duale oder vollzeitschulische berufliche Grundbildung besteht die Möglichkeit, mit dem Besuch eines vor allem auf Allgemeinbildung ausgerichteten Unterrichts den Fachhochschulzugang erwerben. Schüler vollschulischer Berufsbildungen, die den Fachhochschulzugang erlangen wollen, müssen zusätzlich ein betriebliches Praktikum absolvieren.

Abschließend lässt sich festhalten, dass sich duale Berufsausbildungen durch einen höheren Anteil berufsspezifischer Qualifikationen und vollschulische Berufsausbildungen durch einen höheren Anteil allgemeiner Qualifikationen auszeichnen. Bei beiden Ausbildungsformen variieren der Umfang der Qualifikationen mit der Dauer und die Komplexität der Qualifikationen mit dem Anforderungsniveau des Ausbildungsgangs. Umfang und Komplexität der erworbenen Qualifikationen alleine dürften jedoch in einem segmentierten Arbeitsmarkt einen fachadäquaten Berufseinstieg nicht entscheidend prägen, da jede zertifizierte Ausbildung ein bestimmtes Qualifikationsbündel vermittelt, das den Zugang zu einem entsprechenden fachlichen Segment auf dem Arbeitsmarkt bietet. Zu erwarten ist aber, dass das Arbeitslosigkeitsrisiko bei tieferer Qualifikationsausstattung steigt und die Berufswechselneigung bei umfangreicherer Qualifikationsausstattung sinkt.

\subsection{Die fachliche Zusammensetzung des Stellenangebots und die Berufseinstiegschancen}

Die beschriebenen Eigenheiten des berufsorientierten Bildungssystems führen dazu, dass fachspezifische Beschäftigungsmöglichkeiten den Berufseinstieg mitbestimmen. Gemäß dem Labour-Queue-Ansatz (Thurow 1975, 1978) reihen die Arbeitgeber die Stellenanwärter anhand der erwarteten Einarbeitungskosten in einer Arbeitskräfteschlange ein und berücksichtigen die zuerst positionierte Person. Die Signaling-Theorie (Spence 1973) ergänzt diesen Ansatz durch die Annahme, dass die Arbeitgeber die Einarbeitungskosten und die Produktivität nicht direkt beobachten können. Sie stützen sich deshalb bei der Personalauswahl auf bestimmte Signale, die in ihren Augen mit der fehlenden Information korrelieren. In berufsorientierten Ausbildungssystemen mit einhergehender Segmentierung des Arbeitsmarkts entlang fachlicher Differenzierungslinien figurieren fachspezifische Ausbildungszertifikate als primäres Signal für die Einarbeitungskosten. Die Firmen reihen Absolventen mit einem fachlich der Stelle entsprechenden Zertifikat weiter vorne in der Arbeitskräfteschlange ein als Absolventen mit fachfremden Zertifikaten. Somit erhöht beim Berufseinstieg in erster Linie das facheigene Stellenangebot und nicht die Zahl der offenen Stellen im Gesamtarbeitsmarkt die Beschäftigungsmöglichkeiten.

Der Labour-Queue-Ansatz (Thurow 1975, 1978) postuliert weiter, dass bei einem Angebotsüberhang die Arbeitgeber vermehrt zwischen den Bewerbern auswählen können. Sie erhöhen dann ihre Anforderungen, womit Signale als Einreihungskriterium an Bedeutung gewinnen. Bei einem Nachfrageüberhang müssen die Arbeitgeber dagegen die Anforderungen herabsetzen. Sie stellen dann wohl auch Arbeitskräfte ein, die einen fachfremden Beruf erlernt haben. Für die Berufseinsteiger bieten also auch fachfremde Stellenangebote, die qualifikationsmässig nicht der beruflichen Aus- 
bildung entsprechen, Beschäftigungsmöglichkeiten, falls im betreffenden Berufssegment ein Nachfrageüberhang besteht.

Neben der fachlichen Ausrichtung der Ausbildung schränkt die mangelnde Arbeitsmarkterfahrung die Beschäftigungsmöglichkeiten von Berufseinsteigern ein. Arbeitgeber betrachten für einen Großteil der ausgeschriebenen Stellen Arbeitsmarkterfahrung als entscheidendes Einstellungskriterium (Salvisberg und Sacchi 2014), da sie diese als Signal für niedrige Einarbeitungskosten werten. Somit können für Berufseinsteiger in segmentierten Arbeitsmärkten nur jene offenen Stellen als individuell passend bezeichnet werden, die fachlich dem Ausbildungsberuf entsprechen und keine Arbeitsmarkterfahrung voraussetzen.

Erfahrene Arbeitskräfte können sich dagegen auf Stellenausschreibungen bewerben, die Berufserfahrung voraussetzen. Sie finden aufgrund von unter Umständen fachlich diversifizierten Arbeitserfahrungen und Weiterbildungen auch Beschäftigungsmöglichkeiten außerhalb des erlernten Berufs im gesamten Arbeitsmarkt. Wenn die Nachfrage nach Arbeitskräften insgesamt jedoch niedrig ist, konkurrieren sie Berufseinsteiger vermehrt um Einsteigerstellen. Das Gesamtstellenangebot bildet also die Konkurrenz auf dem Gesamtarbeitsmarkt ab und bestimmt darüber, ob Berufseinsteiger von erfahrenen Arbeitskräften auf die hinteren Plätze der Arbeitskräfteschlange verwiesen werden. Gibt es ebenfalls nur wenige für Einsteiger geeignete offene Stellen im erlernten Beruf, verdrängen sie möglicherweie Berufseinsteiger aus dem Beruf. Diese müssen dann Berufswechsel oder Arbeitslosigkeit in Kauf nehmen.

Aus diesen theoretischen Überlegungen leiten wir vier Hypothesen ab:

H1: Je umfangreicher das Angebot an passenden Stellen ist, desto höher ist die Wahrscheinlichkeit einer fachlich adäquaten Beschäftigung. Je niedriger jedoch die Zahl der offenen Stellen im erlernten Beruf ist, desto eher müssen Berufseinsteiger Berufswechsel oder Arbeitslosigkeit in Kauf nehmen.

H2: Je umfangreicher das fachfremde Stellenangebot ist, desto höher ist die Berufswechselneigung.

H3: Die Stärke der Arbeitsnachfrage auf dem Gesamtarbeitsmarkt allein beeinflusst den Berufseinstieg nicht.

H4: Je niedriger die Personalnachfrage insgesamt ist, desto stärker erhöht eine abnehmende Zahl passender offener Stellen die Berufswechselneigung und das Arbeitslosigkeitsrisiko.

\subsection{Der Berufseinstieg im Zusammenspiel von Qualifikationsausstattung und Qualifikationsnachfrage}

Wie die Erläuterungen in Abschn. 2.1 gezeigt haben, unterscheiden sich die beruflichen Grundbildungen nicht nur in fachlicher Hinsicht, sondern steuern auch durch die unterschiedliche Qualifikationsausstattung den Zugang zum Arbeitsmarkt. Dabei dürften insbesondere Unterschiede im Anteil der vermittelten berufsspezifischen und allgemeinen Qualifikationen in dualen und vollschulischen Berufsausbildungen bedeutsam sein. Humankapitalansätze (Becker 1964; Mincer 1974) gehen davon aus, dass die durch Bildung erworbenen Qualifikationen berufsspezifisch oder allgemein sein können (Dust- 
mann und Meghir 2005). Berufsspezifische Qualifikationen lassen sich nur schwerlich in andere Berufe transferieren. Allgemeine Qualifikationen erlauben eine höhere fachliche Mobilität auf dem Arbeitsmarkt. Weiter gehen Humankapitalansätze als individuelle Handlungstheorien davon aus, dass Individuen immer nach der besten Verwertung ihrer Qualifikationen auf dem Arbeitsmarkt streben. Berufswechsel führen aufgrund eingeschränkter Transfermöglichkeiten berufsspezifischer Qualifikationen zu einer Entwertung des berufsspezifischen Humankapitals. Berufseinsteiger bevorzugen also grundsätzlich eine Stelle im erlernten Beruf. Es ist zudem davon auszugehen, dass dies umso mehr der Fall ist, je größer der Umfang der erworbenen berufsspezifischen Qualifikationen ist. Berufswechsel streben Berufseinsteiger nur an, wenn sie die (längerfristigen) Vorteile einer fachfremden Stelle höher einstufen als die drohende Humankapitalentwertung bei Verlassen des erlernten Berufs.

Auch wenn die Absolventen beruflicher Grundbildungen Berufswechsel nicht intendieren, sind sie unter Bedingungen geringer fachlich passender Beschäftigungsmöglichkeiten oft nicht zu vermeiden, um einer länger andauernden Phase von Arbeitslosigkeit zu entgehen. Arbeitssuchende Personen können innerhalb der zugänglichen offenen Stellen die aktuell beste (verfügbare) auswählen oder ihre Suchbemühungen fortsetzen (Mortensen 1986; Rogerson et al. 2005). Je umfangreicher die in der Ausbildung erworbenen allgemeinen Qualifikationen sind, desto größer sind die bei einem Berufswechsel transferierbaren Qualifikationen, die bei einer weiteren Suche und somit Arbeitslosigkeit nicht verwertet werden könnten. Somit steigen die Kosten der Stellensuche gegenüber einem Berufswechsel. Je umfangreicher dagegen die in der Ausbildung erworbenen beruflichen Qualifikationen sind, desto größer sind die bei einem Berufswechsel entwerteten Qualifikationen. Somit übertreffen die Kosten eines Berufswechsels eher diejenigen der Stellensuche.

Die Überlegungen zur individuell bestmöglichen Verwertung der in der Ausbildung erworbenen Qualifikationen lassen sich mit den in Abschn. 2.2 beschriebenen Gedanken zu nachfrageseitigen Faktoren verbinden. So hängen die Chancen der Absolventen, eine der Ausbildung fachlich adäquate Stelle zu erhalten, von den Einstellungsentscheidungen der Arbeitgeber ab. Arbeitgeber dürften im Sinn der Signaling-Theorie berufliche Spezialisierung, betriebliche Sozialisation und Praxiserfahrung für eine fachadäquate Beschäftigung positiv werten. Somit verfügen Absolventen dualer Berufsausbildungen über bessere Beschäftigungschancen im erlernten Beruf als vollschulisch Ausgebildete, insbesondere wenn letztere keinen Fachhochschulzugang erworben und somit kein Praktikum absolviert haben. Jedoch spielen die Signale berufliche Spezialisierung, betriebliche Sozialisation und Praxiserfahrung je nach Stärke der fachspezifischen Qualifikationsnachfrage eine mehr oder weniger bedeutende Rolle. Vor allem bei einem schwachen Personalbedarf dienen sie den Arbeitgebern für die Auswahl.

Vor dem Hintergrund dieser theoretischen Überlegungen können die folgenden drei Hypothesen aufgestellt werden:

H5: Je höher der Anteil der erworbenen berufsspezifischen Qualifikationen, desto niedriger ist die Berufswechselneigung. 
H6: Je geringer das passende Stellenangebot, desto eher nehmen Berufseinsteiger mit einem hohen Anteil berufsspezifischer Qualifikationen Arbeitslosigkeit in Kauf, während Berufseinsteiger mit einem hohen Anteil allgemeiner Qualifikationen eher den Beruf wechseln.

H7: Je geringer das passende Stellenangebot, desto eher erhalten Absolventen mit Praxiserfahrung, betrieblicher Sozialisation und beruflicher Spezialisierung eine ausbildungsadäquate Beschäftigung als Absolventen ohne diese Merkmale.

\subsection{Individuelle Merkmale im Verbund mit der fachspezifischen}

Qualifikationsnachfrage: Die gemeinsame Bedeutung für den Berufseinstieg

Neben Merkmalen der beruflichen Grundbildung beachten die Arbeitgeber bei der Einstellung von neuen Mitarbeitenden auch individuelle Merkmale als Signale für die Produktivität. Dazu zählen das Geschlecht, ein Migrationshintergrund und Abschlussnoten. Die letzteren dienen als Indikator für kognitive Fähigkeiten. Stereotype Annahmen und negative Zuschreibungen führen dazu, dass Arbeitgeber Frauen und Personen mit einem Migrationshintergrund oft eine niedrigere Produktivität unterstellen (Bielby und Baron 1986; Reskin und Roos 1990). Geschlecht, Migrationshintergrund und kognitive Fähigkeiten bestimmen aber bereits die Berufswahl (Bertschy et al. 2008) und Lehrlingsrekrutierung (Müller und Wolter 2013). Es ist belegt, dass der Erwerb eines bestimmten Zertifikats ein stärkeres Signal für die Produktivität darstellt als individuelle Merkmale (z. B. Bertschy et al. 2008; Müller und Schweri 2009). Welche Signale bei einem geringen Personalbedarf den Berufseinstieg steuern, ist bislang jedoch unklar. Da gemäß der Labour-Queue-Theorie Signale bei einem Angebotsüberhang an Bedeutung gewinnen, dürften bei einer niedrigen Anzahl offener Stellen neben den Zertifikaten auch individuelle Merkmale den Berufseintritt beeinflussen. Wenn Arbeitgeber aufgrund eines berufsspezifischen Angebotsüberhangs zwischen Arbeitskräften mit denselben Zertifikaten aussuchen können, verbleiben individuelle Merkmale als Auswahlkriterium.

H8: Je schlechter das passende Stellenangebot ist, desto höher ist die Wahrscheinlichkeit eines Berufswechsels oder Arbeitslosigkeit von Frauen und Personen mit Migrationshintergrund oder tiefen Abschlussnoten im Vergleich zu Männern, Inländern oder Personen mit guten Noten.

Berufswechsel beim Arbeitsmarkteintritt als Folge individueller Eigenschaften können auch aus psychologisch-entscheidungstheoretischer Sicht erklärt werden. Die Passungstheorie (Holland 1959) und der Self-enhancement-Ansatz (Pekrun 1987) postulieren, dass die Passung von persönlichen Charakteristiken mit der Berufswahl die nachfolgend erbrachten Leistungen beeinflusst (Gerber-Schenk et al. 2010). Eine hohe Passung, ausgedrückt in höherer Zufriedenheit mit der Ausbildung, führt demnach nach Lehrabschluss zu einer intensiveren Stellensuche im erlernten Beruf als eine schlechte Passung. Und schlussendlich erhalten Berufseinsteiger mit einer hohen Passung eher eine der Ausbildung entsprechende Beschäftigung und wechseln seltener den Beruf oder sind arbeitslos. Eine schlechte Passung dagegen steigert die Motivation für einen Berufswechsel. Die Passung dürfte vor allem bei einer 
geringen Zahl an facheigenen offenen Stellen entscheidend sein. Mit dem erlernten Beruf unzufriedene Personen, die nicht intensiv nach einer Stelle im erlernten Beruf suchen, werden dann eher aus dem Beruf verdrängt. Zudem suchen sie aufgrund der geringen Beschäftigungsmöglichkeiten im erlernten Beruf auch vermehrt nach einer fachfremden Tätigkeit.

H9: Je geringer das passende Stellenangebot ist, desto eher wechseln mit dem erlernten Beruf unzufriedene im Vergleich zu zufriedenen Personen den Beruf.

Insgesamt implizieren die theoretischen Ansätze, dass in einem berufsfachlich segmentierten Arbeitsmarkt das Volumen des passenden sowie des fachfremden Stellenangebots die fachliche Adäquanz des Berufseinstiegs determiniert. Die Zahl der offenen Stellen im erlernten Beruf entscheidet insbesondere auch über die Verwertbarkeit von beruflichen Qualifikationen und den Einfluss individueller Merkmale. Im Folgenden stellen wir die Datenbasis vor, anhand welcher wir die formulierten Hypothesen überprüfen.

\section{Daten und Methoden}

Als Datengrundlage für die Analysen dient das Schweizer Jugendpanel PISA2000/ TREE $^{1}$. Es zeichnet den nachobligatorischen Ausbildungsverlauf und den Berufseinstieg von Jugendlichen nach, welche im Jahr 2000 an der ersten PISA-Befragung teilgenommen und im selben Jahr die obligatorische Schulzeit beendet haben. Zwischen 2001 und 2007 wurde die ursprüngliche Stichprobe von rund 6000 Personen im Jahresrhythmus nachbefragt, wobei im Jahr 2007 noch knapp 4000 Antworten realisiert wurden (TREE 2011). Wir berücksichtigen Jugendliche mit Abschluss einer beruflichen Grundbildung in den Jahren 2002 bis 2005, die jeweils ein Jahr nach Abschluss der Ausbildung erwerbstätig sind oder eine Stelle suchen. Für die Auswertungen stehen uns nach Ausschluss von Fällen ohne Berufsangaben 1358 Lehrabgänger zur Verfügung. Um Verzerrungen durch Stichprobenausfälle zu kompensieren, haben wir Längsschnittgewichtungen für den jeweils individuellen Berufseinstiegszeitpunkt, d. h. die letzte von uns verwendete Welle, berechnet und unsere Auswertungen damit gewichtet (vgl. Sacchi 2008). ${ }^{2}$

Berufswechsel messen wir als Wechsel zwischen den Berufsklassen (2-Steller mit 39 Berufskategorien) der Schweizerischen Berufsnomenklatur SBN2000. Hierfür wurden die Ausbildungsberufe, die ursprünglich nach dem für Ausbildungen angelegten BIS (Bildungsstatistisches Informationssystem) des Bundesamts für Statistik codiert waren, in die Schweizerische Berufsnomenklatur transformiert. Dabei wurde anhand der Textangaben überprüft, dass gleiche Tätigkeiten, die unterschiedlich

\footnotetext{
${ }^{1}$ Die Schweizer Jugendlängsschnittstudie TREE (Transitionen von der Erstausbildung ins Erwerbsleben, www.tree.unibas.ch) läuft seit 2000 und wurde bisher durch den Schweizerischen Nationalfonds, die Universität Basel, die Bundesämter für Berufsbildung und Technologie und Statistik sowie die Kantone Bern, Genf und Tessin finanziert.

${ }^{2}$ Fehlende Werte in den erklärenden Variablen „Abschlussnoten“ und „Zufriedenheit“ haben wir mittels einfacher Mittelwertimputation imputiert.
} 
codiert werden können, nicht als Berufswechsel gewertet sind (s. Müller und Schweri 2009; Müller und Schweri 2015). Aufgrund dieser Maßnahme und der relativ breit gefassten Definition von Berufswechseln dürften die Verzerrungen schwach ausfallen, die bei einem Vergleich von Ausbildungen mit Berufen über Nomenklaturen normalerweise entstehen.

Die Stärke der Qualifikationsnachfrage messen wir anhand von Stelleninseratedaten des Stellenmarktmonitors-Schweiz (www.stellenmarktmonitor.ch; Sacchi et al. 2005). Die Daten beinhalten jährliche repräsentative Stichproben von knapp 5000 Stellenanzeigen in Presse und Internet in der Schweiz. Die Messreihe reicht bis 1950 zurück und wird laufend fortgeschrieben. ${ }^{3}$ Die Daten der offenen Stellen erlauben dank der detaillierten Erfassung von Stellen- und Firmenmerkmalen sowie den Anforderungen an die Bewerbenden eine differenzierte Analyse der Nachfrage nach Arbeitskräften mit spezifischen beruflichen Qualifikationen (Kriesi et al. 2010). Da die Betriebe umso mehr Stellenanzeigen aufgeben, je schwieriger sich die Suche nach geeigneten Arbeitskräften gestaltet, bilden die Daten der Stellenanzeigen annäherungsweise den Nachfrageüberhang ab. Mit diesen Daten können wir berufsspezifische Beschäftigungsmöglichkeiten auf der Mikroebene des individuellen Berufseinsteigers messen und müssen uns nicht wie die bisherige Forschung auf aggregierte Makrodaten wie die allgemeine (oder regionale) Arbeitslosenquote oder die jährliche Veränderung des BIB stützen. Wir verknüpfen dazu die Daten der offenen Stellen auf Berufsebene, zeitpunktspezifisch und regional mit den TREE-Individualdaten entsprechend den Angaben für jeden Berufseinsteiger. ${ }^{4}$ Es werden drei Indikatoren gebildet: Das aufgrund der erworbenen Qualifikationen passende, das fachfremde und das Gesamtstellenangebot.

Das passende Stellenangebot entspricht den für Berufseinsteiger aufgrund der fachlichen Ausrichtung ihrer Ausbildung und der mangelnden Berufserfahrung zugänglichen freien Stellen im Jahr nach Ausbildungsabschluss in der Sprachregion, in welcher die Jugendlichen wohnen. ${ }^{5}$ Wir zählen in Anlehnung an Sacchi und Salvisberg (2012) jene Anzeigen als Einsteigerstellen, die sich an Fachkräfte mit beruflicher Grundbildung richten und weder Erfahrung noch Weiterbildung voraussetzen, die keine Leitungsfunktion beinhalten und die Berufseinsteiger im Inseratetext auch nicht aufgrund ihres jugendlichen Alters ausschließen. Da die fachliche Segmentierung des Arbeitsmarkts nie absolut ist, existieren in Realität immer Zertifikate, welche zu verschiedenen Berufen Zugang bieten. Solche Zugangsmöglichkeiten berücksichtigen wir über folgendes Verfahren: Die über die Berufe hochgerechneten Stellen-

\footnotetext{
${ }^{3}$ Für genauere Informationen zum Datensatz und Erhebungsdesign siehe www.stellenmarktmonitor.uzh. ch; für die externe Validierung der Daten siehe Sacchi et al. (2005). Sacchi et al. (2005) weisen nach, dass die erhobenen Stellenanzeigen den gesamten Arbeitsmarkt abdecken. Die Ergebnisse von Sacchi und Salvisberg (2014) lassen zudem erkennen, dass die Daten der Stellenanzeigen die Nachfrage in den verschiedenen Berufen angemessen abbilden.

${ }^{4}$ Dieses Vorgehen hat sich unter anderem bei der Untersuchung von Mobilitätsprozessen im Arbeitsmarkt bereits als fruchtbar erwiesen (siehe z. B.: Buchmann et al. 2009; Kriesi et al. 2010; Kriesi 2006).

${ }^{5}$ Tessin und französischsprachige Schweiz fassen wir zusammen. Vor dem Hintergrund der Kleinräumigkeit der Schweiz nehmen wir keine weitere regionale Differenzierung vor und gehen davon aus, dass Berufseinsteiger, die geografisch noch wenig gebunden sind, grundsätzlich in der gesamten Sprachregion nach Stellen suchen.
} 
anzeigen gewichten wir anhand von Übergangswahrscheinlichkeiten vom jeweils erlernten in den in der Anzeige erwähnten Beruf. Konkret benutzen wir AusbildungsBeruf-Matrixen von 18 bis 25-jährigen Personen mit beruflicher Grundbildung aus der Schweizer Volkszählung 2000. Je kleiner die Übergangswahrscheinlichkeit ist, desto schwächer werden die betroffenen Stellenanzeigen gewichtet. Dazu verwenden wir folgende Formel:

$$
O_{a j}=\sum_{b=1}^{B}\left(w_{a b} * n_{b j}\right)
$$

$\mathrm{O}_{\text {aj }} \quad$ Anzahl offene Stellen in Jahr j gewichtet mit den individuellen Zugangschancen einer Person mit der Ausbildung a.

$\mathrm{w}_{\mathrm{ab}}$ Übergangsrate von Ausbildung $\mathrm{a}$ in Beruf $\mathrm{b}$.

$\mathrm{n}_{\mathrm{bj}} \quad$ Anzahl an im Jahr j ausgeschriebenen Stellen mit dem auszuübenden Beruf $\mathrm{b}$.

Das fachfremde Stellenangebot bildet die Beschäftigungsmöglichkeiten im Einstiegsjahr für Personen mit beruflicher Grundbildung in den fachlich entfernteren Berufen in der Sprachregion ab. Für diesen Indikator summieren wir die hochgerechneten und nach obigem Verfahren gewichteten Stellenanzeigen über alle Berufe, außer den erlernten. Um auszuschließen, dass gut zugängliche, aber qualifikatorisch entfernte offene Stellen diesen Indikator zu stark beeinflussen, summieren wir nur Stellen anderer 1-Steller-Berufe der Schweizer Berufsnomenklatur.

Das Gesamtstellenangebot umfasst das Volumen der zum Einstiegszeitpunkt insgesamt ausgeschriebenen offenen Stellen für Personen mit beruflicher Grundbildung in der Sprachregion. Es soll die Beschäftigungsmöglichkeiten für alle Arbeitskräfte, also auch solche mit Berufserfahrung, erfassen. Somit ist es auch ein Indikator für die Konkurrenz, der Berufseinsteiger ausgesetzt sind.

Der über die berufliche Grundbildung vermittelte Anteil beruflicher und allgemeiner Qualifikationen wird über den Ausbildungstyp gemessen. Der Ausbildungstyp unterscheidet duale und vollzeitberufliche Ausbildungsformen sowie jeweils den Erwerb des Fachhochschulzugangs. Die Ausbildungsdauer ist ein Indikator für den Umfang der jeweiligen Qualifikationen. Das Anforderungsniveau der Ausbildung ist Resultat eines auf die geforderten intellektuellen Fähigkeiten fokussierten Expertenratings von Berufslehrern (Stalder 2011). Weil kaufmännische Ausbildungen mit Abstand die am häufigsten gewählten Berufsausbildungen sind und auch die meisten vollschulischen Ausbildungen in diesem Ausbildungsberuf stattfinden, schließen wir eine Kontrollvariable dafür in die Analysen ein. Ebenso kontrollieren wir die Sprachregion, da sich der Anteil dualer und vollschulischer Ausbildungen zwischen den Regionen aus historischen Gründen unterscheidet (Stalder und Nägele 2011).

Die kognitiven Fähigkeiten messen wir über die für die Arbeitgeber gut sichtbaren Abschlussnoten der Berufsausbildung. Als Kontrolle für nicht sichtbare kognitive Fähigkeiten, welche bereits die Berufswahl beeinflusst haben dürften, verwenden wir die gemessene Lesekompetenz zum Zeitpunkt der PISA-Erhebung im Jahr 2000. Weiter werden das Geschlecht und ein Migrationshintergrund in die Analysen einbezogen. Für die aus passungstheoretischer Sicht bedeutenden Motivationen betrachten wir die Zufriedenheit mit der Ausbildung vor deren Abschluss. 
Tab. 1 Verteilung der Berufseinstiegsalternativen und des Stellenangebots

\begin{tabular}{llll}
\hline & Arbeit im Beruf & Berufswechsel & Arbeitslos \\
\hline $\begin{array}{l}\text { Anteile in Prozent (gewichtet) } \\
\begin{array}{l}\text { Mean (Std. Err.) Stellenangebot für Einsteiger } \\
\text { im Beruf }\end{array}\end{array}$ & $0,067(0,061)$ & 0,08 & 0,09 \\
$\begin{array}{l}\text { Mean (Std. Err.) Stellenangebot in fachfrem- } \\
\text { den Berufen }\end{array}$ & $0,057(0,058)$ & $0,094(0,095)$ & $-0,324(0,123)$ \\
\hline
\end{tabular}

Für eine bessere Vergleichbarkeit wurde das Stellenangebot standardisiert (Mean 0; Std Err. 1)

Mit diesen Indikatoren berechnen wir ein multinomiales logistisches Entscheidungsmodell (Long und Freese 2006). Für eine bessere Interpretierbarkeit der Ergebnisse stellen wir durchschnittliche Marginaleffekte dar. Dies bedeutet, dass die Effekte einzelner Variablen auf die Wahrscheinlichkeit der Wahl der jeweiligen Alternative berechnet werden (Williams 2012).

\section{Ergebnisse}

Gemäß unseren Ergebnissen (Tab. 1) arbeiten rund 83\% der Jugendlichen knapp ein Jahr nach Ausbildungsabschluss im erlernten Beruf, $8 \%$ haben den Beruf gewechselt und gut $9 \%$ sind arbeitslos. ${ }^{6}$ Der größte Teil der Absolventen beruflicher Grundbildungen kann also beim Arbeitsmarkteinstieg den erlernten Beruf ausüben.

Das passende und das fachfremde Stellenangebot verteilen sich jedoch ungleich über die Berufseinstiegalternativen. Absolventen beruflicher Grundbildungen finden eher eine fachadäquate Beschäftigung, wenn die Zahl der Vakanzen für Berufseinsteiger im von ihnen erlernten Beruf überdurchschnittlich hoch ist. Das durchschnittliche Stellenangebot für Jugendliche, die einen Berufswechsel vollzogen haben, ist dagegen im erlernten Beruf tief und in den fachfremden Berufen hoch. Arbeitslosigkeit geht mit tiefen Beschäftigungsmöglichkeiten in beiden Bereichen einher. Diese Verteilungen sind erste Hinweise dafür, dass Umfang und Struktur der Qualifikationsnachfrage eine Rolle beim Berufseinstieg spielen. ${ }^{7}$

Welche Bedeutung die Qualifikationsnachfrage im Vergleich zu Ausbildungsmerkmalen und individuellen Eigenschaften der Berufseinsteiger für die drei Berufseinstiegsalternativen Arbeit im Beruf, Berufswechsel und Arbeitslosigkeit knapp ein Jahr nach Ausbildungsabschluss hat, wird in Abschn. 4.1 dargestellt. Danach werden die Resultate zur Wirkungsweise dieser Faktoren bei unterschiedlich starker Nachfrage nach berufsspezifischen Qualifikationen präsentiert (4.2.).

\footnotetext{
${ }^{6}$ Die Berufseinstiegsalternativen sind ungleich verteilt und die Fallzahlen für Berufswechsel und Arbeitslosigkeit entsprechend eher klein. Jedoch ist die Besetzung in allen Variablenkategorien genügend groß für multivariate statistische Auswertungen. Es werden aber meist nur starke Effekte statistisch signifikant.

${ }^{7}$ Deskriptive Auswertungen zu den weiteren Variablen befinden sich in Tab. 3.
} 
Tab. 2 Multinomiales Logit für die Berufseinstiegsalternativen ein Jahr nach Ausbildungsabschluss

\begin{tabular}{llll}
\hline & \multicolumn{2}{c}{ Marginaleffekte } & \\
\cline { 2 - 4 } Variable & Arbeit im Beruf & Berufswechsel & Arbeitslos \\
\hline Stellenangebot (pro 1000 Stellen) & & & \\
Passend & $0,34^{* *}$ & $-0,24^{* *}$ & $-0,09$ \\
Fachfremd & $-0,04^{*}$ & $0,04^{*}$ & 0,00 \\
Gesamt & 0,04 & $-0,03$ & $-0,01$ \\
Ausbildungstyp (Referenz: Dual ohne FHZ) & & \\
Dual mit FHZ & $-0,01$ & 0,01 & 0,01 \\
Schulisch ohne FHZ & $-0,38^{* *}$ & $0,23^{*}$ & $0,15^{* *}$ \\
Schulisch mit FHZ & $-0,21^{*}$ & $0,19^{*}$ & 0,02 \\
Ausbildungsdauer (Referenz: 3 Jahre) & & \\
2 Jahre & $-0,05$ & $-0,03$ & 0,08 \\
4 Jahre & 0,01 & $-0,04$ & 0,03 \\
Anforderungsniveau hoch & 0,11 & $-0,04$ & $-0,07$ \\
Frau & 0,06 & $-0,01$ & $-0,05$ \\
Migrationshintergrund & $-0,01$ & 0,00 & 0,01 \\
Lesekompetenzen PISA & $-0,23$ & $-0,11$ & 0,34 \\
Abschlussnote & 0,08 & $-0,02$ & $-0,06$ \\
Mit Ausbildung zufrieden & $0,03^{* *}$ & $-0,02$ & $-0,02^{*}$ \\
Romandie/Tessin & 0,06 & $-0,04$ & $-0,03$ \\
KV & $-0,12$ & 0,04 & 0,08 \\
N & 1358 & & \\
\hline FHZFachhochs & & \\
\hline
\end{tabular}

FHZ Fachhochschulzugang

$* p<0,05 ; * * \mathrm{p}<0,01$

4.1 Arbeit im Beruf, Berufswechsel oder Arbeitslosigkeit nach der Lehre: Die Rolle des Stellenangebots, der Berufsausbildung und individueller Merkmale

In einem fachlich segmentierten und stark auf fachliche Ausbildungszertifikate fokussierten Arbeitsmarkt dürften gemäß unseren Erwartungen vor allem das facheigene und das fachfremde Stellenangebot sowie der jeweilige Anteil der in der Berufsausbildung erworbenen berufsspezifischen und allgemeinen Qualifikationen die Berufseinstiegsalternativen beeinflussen.

Die Ergebnisse in Tab. 2 bestätigen diese Erwartungen. Je umfangreicher das passende Stellenangebot ist, desto eher arbeiten die Jugendlichen im erlernten Beruf und desto seltener werden Berufswechsel (Hypothese H1). Überraschenderweise tritt jedoch Arbeitslosigkeit bei einer schwachen fachspezifischen Arbeitskräftenachfrage nicht häufiger auf, als wenn diese stark ist. Dennoch unterstreicht der Befund, dass in einem Arbeitsmarkt, in welchem fachliche Ausbildungszertifikate eine hohe Signalwirkung für die Einarbeitungskosten besitzen, die für Berufseinsteiger individuell gemessenen facheigenen Beschäftigungsmöglichkeiten den Berufseinstieg bestimmen. Im Einklang mit der relativ tiefen Berufswechselrate bekräftigt die große Bedeutung der Qualifikationsnachfrage im erlernten Beruf die These eines ausgeprägt fachlich segmentierten Arbeitsmarkts in der Schweiz. In einem solchen Arbeitsmarkt sind die Zugangschancen zu Stellen außerhalb des erlernten Berufs klein, steigen 
jedoch mit dem Volumen des fachfremden Stellenangebots. Damit bestätigt sich Hypothese H2. Gleichzeitig sinkt mit Zunahme der fachlich entfernten Beschäftigungsmöglichkeiten die Wahrscheinlichkeit einer fachadäquaten Beschäftigung. Wenn überhaupt, dann stellen die Betriebe also vor allem bei einem berufsspezifischen Nachfrageüberhang auch einmal Arbeitskräfte mit einem fachfremden Ausbildungszertifikat ein. Die kleine Auswahlmöglichkeit zwischen den Bewerbenden zwingt sie anscheinend dazu, die Anforderungen bezüglich fachlicher Ausrichtung der Zertifikate zu reduzieren, was den aus der Labour-Queue-Theorie abgeleiteten Erwartungen entspricht. Im Einklang mit Hypothese $H 3$ hat das Gesamtstellenangebot, das die Konkurrenz auf dem Gesamtarbeitsmarkt abbildet, keinen signifikanten Einfluss auf den Arbeitsmarkteinstieg, wenn mikroanalytisch die beruflich differenzierte Qualifikationsnachfrage berücksichtigt wird. Inwiefern jedoch das Gesamtstellenangebot die Wirkungsweise der fachspezifischen Qualifikationsnachfrage auf den Berufseinstieg steuert (Hypothese H4), wird in Abschn. 4.2.1 diskutiert.

Von den ausbildungsbezogenen Merkmalen beeinflusst nur der Ausbildungstyp und somit der jeweilige Anteil der erworbenen allgemeinen und berufsspezifischen Qualifikationen, sowie Praxiserfahrung und betriebliche Sozialisation den Berufseinstieg. Absolventen vollschulischer Berufsausbildungen arbeiten signifikant seltener im erlernten Beruf und haben eine größere Berufswechselneigung als Absolventen dualer Berufsausbildungen. Haben sie keinen Fachhochschulzugang erworben, sind sie zudem häufiger arbeitslos als dual Ausgebildete. Bei einem hohen Anteil allgemeiner Qualifikationen scheinen Berufswechsel also mit schwächeren Verlusten verbunden zu sein als bei einem hohen Anteil berufsspezifischer Qualifikationen, was die aus der Humankapitaltheorie abgeleitete Hypothese $H 5$ stützt. Erwartungsgemäß beeinflussen in einem segmentierten Arbeitsmarkt die Ausbildungsdauer und das Anforderungsniveau der Ausbildung die Wahrscheinlichkeit eines fachadäquaten Berufseinstiegs kaum. Denn jeder berufliche Lehrgang vermittelt ein bestimmtes Qualifikationsbündel und ermöglicht somit den Zugang zum entsprechenden fachlichen Segment auf dem Arbeitsmarkt. Die Resultate weisen aber darauf hin, dass das Arbeitslosigkeitsrisiko bei tieferer Qualifikationsausstattung steigt und die Berufswechselneigung bei umfangreicherer Qualifikationsausstattung sinkt. Allerdings sind die Effekte nicht signifikant, was wohl an den kleinen Fallzahlen für diese Kategorien liegt. Somit kann Hypothese $H 6$ nicht angenommen werden.

Im Vergleich $\mathrm{zu}$ fachspezifischen Zertifikaten stellen individuelle Merkmale unseren Erwartungen zufolge schwache Signale für die erwartete Produktivität dar und dürften die Eintrittschancen nur wenig bestimmen. In der Tat lassen sich keine direkten Effekte von Geschlecht, Migrationshintergrund, Lesekompetenzen und Abschlussnoten feststellen. Ob aber die Stärke der Signalwirkung von individuellen Merkmalen mit dem Ausma $ß$ der fachspezifischen Qualifikationsnachfrage variiert, wird in Abschn. 4.2.3 diskutiert. Die subjektive Einschätzung der Zufriedenheit mit der absolvierten Ausbildung hat einen direkten Einfluss auf den Berufseinstieg. Eine gute Passung von persönlichen Charakteristiken mit der Berufswahl, die sich in der Zufriedenheit mit der beruflichen Ausbildung niederschlägt, erhöht die Wahrscheinlichkeit einer fachadäquaten Arbeit und reduziert jene der Arbeitslosigkeit. Somit scheint die Motivation zum Erfolg bei der Suche nach einer fachadäquaten Stelle beizutragen. 
Insgesamt verdeutlichen die Ergebnisse zum direkten Einfluss angebots- und nachfrageseitiger Faktoren auf die drei Berufseinstiegsalternativen die, auch im Vergleich zu ausbildungsbezogenen und individuellen Merkmalen, große Bedeutung der in der bisherigen Forschung stark vernachlässigten Qualifikationsnachfrage. Im Folgenden stellen wir Ergebnisse dar, die erstmals aufzeigen, wie angebotsseitige Merkmale und die auf der Individualebene gemessene facheigene und fachfremde Qualifikationsnachfrage gemeinsam auf die Art des Arbeitsmarkteinstiegs wirken.

\subsection{Das Zusammenspiel von angebots- und nachfrageseitigen Faktoren beim Berufseinstieg}

Die grundlegende Annahme hinter den in diesem Abschnitt präsentierten Befunden ist, dass erworbene Qualifikationen und individuelle Ressourcen im Zusammenspiel mit der Stärke des Personalbedarfs den Berufseinstieg bestimmen. Für einen segmentierten Arbeitsmarkt ist zunächst aber die Frage zu klären, ob die Bedeutung der passenden offenen Stellen für den Berufseinstieg bei unterschiedlichem Umfang des Gesamtstellenangebots variiert (4.2.1). Anschließend werden die Ergebnisse präsentiert, wie der Typ der beruflichen Ausbildung (4.2.2.) sowie Abschlussnoten und Zufriedenheit mit der Berufsausbildung (4.2.3.) je nach Ausmaß der fachspezifischen Qualifikationsnachfrage die Wahrscheinlichkeit der drei untersuchten Einstiegsalternativen beeinflussen.

\subsubsection{Die Zusammensetzung des Stellenangebots und der Berufseinstieg}

Tiefe Beschäftigungsmöglichkeiten auf dem Gesamtstellenmarkt führen auch in einem segmentierten Arbeitsmarkt zu Verdrängungseffekten von Berufseinsteigern durch erfahrene Arbeitskräfte, weil Berufserfahrung ein entscheidendes Einstellungskriterium darstellt (Salvisberg und Sacchi 2014). Vor diesem Hintergrund ist davon auszugehen, dass die Nachfrage nach Arbeitskräften im Gesamtarbeitsmarkt die Wirkungsweise des passenden Stellenangebots für den Berufseinstieg moderiert.

Abbildung 1 veranschaulicht, dass bei einem großen Gesamtstellenangebot die Berufseinsteiger in der Regel auch bei wenigen facheigenen Vakanzen eine Beschäftigung im erlernten Beruf finden. ${ }^{8}$ Ist es dagegen klein, üben passende Beschäftigungsmöglichkeiten einen starken Einfluss auf die fachliche Adäquanz der Beschäftigung aus. Wenn im Gesamtstellenmarkt nur wenige Stellen offen sind, konkurrieren erfahrene Arbeitskräfte und Berufseinsteiger stärker um Einstiegspositionen. Sind auch diese knapp, müssen Berufseinsteiger vermehrt den Beruf wechseln (Hypothese H4). Diese Verdrängungseffekte entstehen vermutlich, weil Arbeitgeber Berufserfahrung als positives Signal für die Einarbeitungskosten im erlernten Beruf werten und deshalb erfahrende Arbeitskräfte bevorzugen. Dass das Arbeitslosigkeitsrisiko von Berufseinsteigern niedrig bleibt, auch wenn sowohl das Gesamtstellenangebot als

\footnotetext{
${ }^{8}$ Den Berechnungen der Interaktionen liegt jeweils dasselbe Modell wie in Tab. 2 zugrunde, wobei die Interaktionsterme einzeln eingeführt werden. Signifikante Interaktionseffekte werden für eine bessere Interpretierbarkeit hier und in den folgenden Abb. 2, 3, 4 bildlich dargestellt. Eine Tabelle mit allen Interaktionseffekten befindet sich in Tab. 5 .
} 

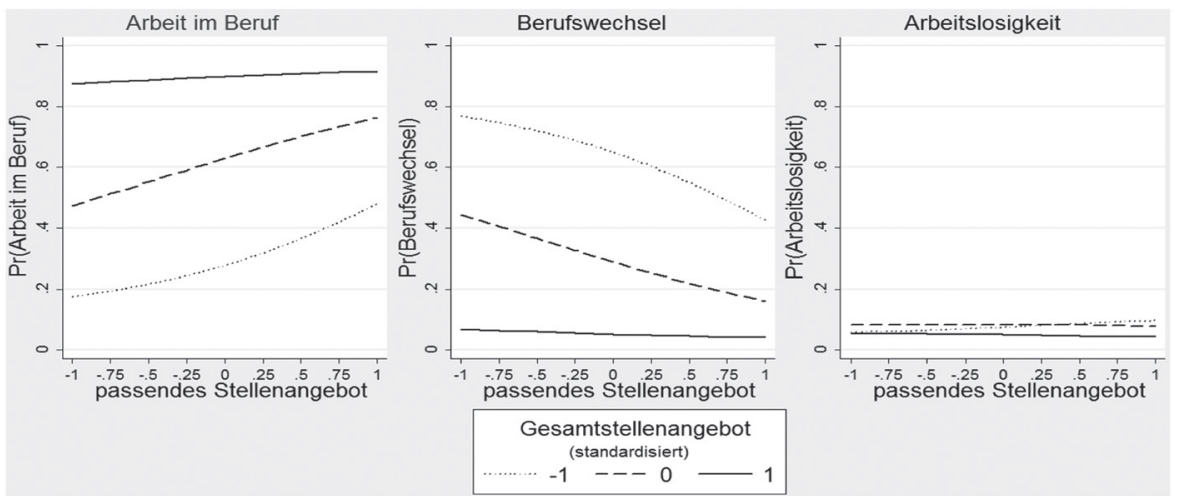

Stellenangebotskategorien sind z-standardisiert; $n=1358$.

Abb. 1 Geschätzte Wahrscheinlichkeit der Berufseinstiegsalternativen für Interaktionen zwischen Stellenangebotskategorien.
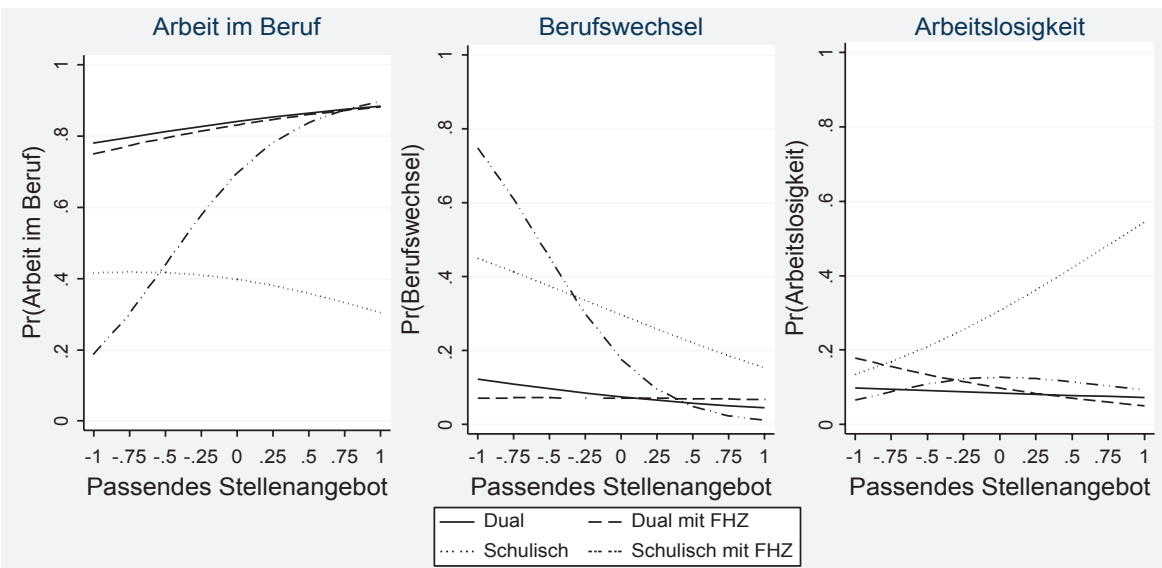

Stellenangebot ist $z$-standardisiert; $n=1358 ; \mathrm{FHZ}=$ Fachhochschulzugang.

Abb. 2 Geschätzte Wahrscheinlichkeit der Berufseinstiegsalternativen nach Ausbildungstyp und Stellenangebot.

auch die Zahl passender offener Stellen niedrig sind, dürfte in der niedrigen Arbeitslosigkeit in der Schweiz begründet sein. Offenbar gelingt es den Absolventen beruflicher Grundbildung, Arbeitslosigkeit zu verhindern, indem sie auf Berufe mit guten Beschäftigungsmöglichkeiten ausweichen.

\subsubsection{Ausbildung und Berufseinstieg bei unterschiedlich starker fachspezifischer Qualifikationsnachfrage}

In den theoretischen Ausführungen haben wir dargelegt, dass sich duale und vollschulische Berufsausbildungen nach ihrem jeweiligen Anteil an berufsspezifischen und allgemeinen Qualifikationen sowie bezüglich betrieblicher Sozialisation und 
Praxiserfahrung unterscheiden. Dies dürfte je nach Umfang des fachspezifischen Stellenangebots zu unterschiedlichen Opportunitäten beim Berufseinstieg führen.

So zeigt Abb. 2, dass die Absolventen dualer Ausbildungen ohne und mit Fachhochschulzugang insgesamt über die besten Arbeitsmarktchancen im Anschluss an die Ausbildung verfügen. Sie sind auch bei geringen offenen Stellen meist fachadäquat beschäftigt und müssen selten den Beruf wechseln oder Arbeitslosigkeit akzeptieren. Betriebliche Sozialisation und Praxiserfahrung in der dualen Berufslehre scheinen positive Signale für die Produktivität im erlernten Beruf darzustellen. Die postulierten Humankapitalverluste aufgrund des hohen Anteils berufsspezifischer Qualifikationen machen Berufswechsel für dual Ausgebildete zudem unattraktiv. Entgegen unseren Erwartungen steigt das Arbeitslosigkeitsrisiko von dual Ausgebildeten jedoch nicht, wenn der Umfang des passenden Stellenangebots sinkt. Der mehrheitlich gelungene Berufseinstieg dual Ausgebildeter dürfte also eine Folge der Verbindung insgesamt sehr guter Beschäftigungsmöglichkeiten am Schweizer Arbeitsmarkt mit fachlicher Spezialisierung, betrieblicher Sozialisation und Praxiserfahrung in den dualen Ausbildungen sein.

Jugendliche mit vollschulischen Berufsausbildungen ohne Fachhochschulzugang arbeiten seltener im erlernten Beruf als dual Ausgebildete. Die Wahrscheinlichkeit einer fachadäquaten Beschäftigung steigt auch nicht, wenn der Umfang an passenden Stellenangeboten wächst. Jedoch steuert die fachspezifische Qualifikationsnachfrage, ob die Absolventen vollschulischer Berufsausbildungen ohne Fachhochschulzugang eher den Beruf wechseln oder eher Arbeitslosigkeit akzeptieren. Arbeitslosigkeit nehmen sie eher in Kauf, wenn die Arbeitsnachfrage im erlernten Beruf groß ist und somit die Suchkosten für eine fachadäquate Beschäftigung klein sind. Die Berufswechselneigung ist dagegen höher, wenn das passende Stellenangebot und somit die Aussichten auf eine fachadäquate Beschäftigung niedrig sind. Der hohe Anteil allgemeiner Qualifikationen macht Berufswechsel in diesem Fall im Vergleich zu Arbeitslosigkeit attraktiv. Jedoch scheinen auch bei vollschulischen Berufsausbildungen die Verluste bei einem Berufswechsel erheblich zu sein, weshalb bei guten Beschäftigungsmöglichkeiten im erlernten Beruf oft auch eine sehr lange Suchdauer von rund einem Jahr in Kauf genommen wird. Zusammen mit den vergleichsweise geringen Chancen auf eine Beschäftigung im erlernten Beruf weisen die langen Suchdauern darauf hin, dass die Betriebe Absolventen beruflicher Grundbildungen ohne Praxiserfahrung oder betriebliche Sozialisation (d. h. vollschulische Berufsausbildungen ohne Fachhochschulzugang) weit hinten in der Arbeitskräfteschlange einordnen. Entgegen unseren Erwartungen sind die Betriebe jedoch auch bei kleiner Bewerberauswahl selten bereit, Absolventen einzustellen, die nicht über diese für die erwartete Produktivität bedeutenden Merkmale verfügen.

Die Absolventen vollschulischer Berufsausbildungen mit Fachhochschulzugang haben bei einem kleinen passenden Stellenangebot eine tiefere Wahrscheinlichkeit, im erlernten Beruf zu arbeiten als dual Ausgebildete. Mit zunehmendem Personalbedarf steigt jedoch die Wahrscheinlichkeit einer fachadäquaten Beschäftigung stark an. Am oberen Ende ziehen sie mit den dual ausgebildeten Lehrabgängern gleich. In Übereinstimmung mit unseren Erwartungen ist die Berufswechselneigung bei wenigen offenen Stellen im erlernten Beruf hoch und sinkt mit zunehmender fachspezifischer Qualifikationsnachfrage signifikant. Bezüglich des Arbeitslosigkeitsrisikos 
unterscheiden sich die Absolventen vollschulischer Ausbildungen mit Fachhochschulzugang nicht von dual Ausgebildeten. Weiter zeigt sich bei vollschulischen Ausbildungen mit Fachhochschulzugang, dass die Signalwirkung von Praxiserfahrung und betrieblicher Sozialisation von der Zahl der facheigenen offenen Stellen abhängig ist. Ist die fachspezifische Arbeitsnachfrage und somit die Auswahlmöglichkeit für die Betriebe klein, besitzen die Absolventen vollschulischer Ausbildungen mit Fachhochschulzugang intakte Chancen auf eine fachadäquate Beschäftigung. Die im Vergleich zu Absolventen vollschulischer Berufsausbildungen ohne Fachhochschulzugang wesentlich höhere Wahrscheinlichkeit einer fachadäquaten Beschäftigung bei vielen passenden offenen Stellen verdanken sie vermutlich dem absolvierten Praktikum.

Insgesamt bestätigen die Ergebnisse unsere Erwartungen zu den je nach Umfang des fachspezifischen Stellenangebots unterschiedlichen Opportunitäten von Absolventen verschiedener Ausbildungstypen beim Arbeitsmarkteintritt (Hypothesen H6 und 47 ). Insbesondere bei knappen fachadäquaten Beschäftigungsmöglichkeiten nach der Lehre entscheidet die Zusammensetzung der erworbenen Qualifikationen sowie Praxiserfahrung und betriebliche Sozialisation über den Berufseinstieg.

\subsubsection{Individuelle Merkmale und passende Vakanzen: Das Zusammenspiel beim Berufseinstieg}

Gemäß den theoretischen Überlegungen sollten individuelle Merkmale bei einer schwachen fachspezifischen Qualifikationsnachfrage die Beschäftigungschancen beeinflussen. Unter diesen Bedingungen ist die Konkurrenz unter den Stellensuchenden groß, da die Betriebe viele Bewerbungen mit den erforderlichen Qualifikationen erhalten. So können sie sich bei der Auswahl stärker auf individuelle Merkmale als Signale für die erwartete Produktivität stützen. Die Resultate zeigen, dass das Geschlecht keinen signifikanten Einfluss auf die drei Berufseinstiegsalternativen hat, wenn das passende Stellenangebot niedrig ist, was in Widerspruch zu Hypothese $H 8$ steht. Vermutlich bewirkt die starke geschlechtsspezifische Segregation der Berufsausbildungen und des Arbeitsmarkts in der Schweiz (Charles und Bradley 2009; Charles 2005), dass Geschlechterstereotypen die Chancen von Frauen nicht reduzieren, da die von ihnen häufig erlernten Berufe ohnehin weiblich konnotiert sind. Um den Zusammenhang von Qualifikationsnachfrage, Geschlecht und Arbeitsmarktsegregation besser zu verstehen, ist allerdings zusätzliche Forschung notwendig. Ein Migrationshintergrund scheint dagegen vor allem bei kleinem Personalbedarf das Arbeitslosigkeitsrisiko zu erhöhen. Der Effekt ist jedoch nicht signifikant, da einerseits die Fallzahlen in dieser Kategorie klein sind und andererseits der Inländervorrang nicht auf alle Nationalitäten zutreffen dürfte, was die Varianz der Schätzung erhöht. Abschlussnoten als weiteres Signal für die erwartete Produktivität spielen dagegen mit dem Personalbedarf im erlernten Beruf zusammen. Die Wahrscheinlichkeit der Berufseinstiegsalternativen für Absolventen beruflicher Grundbildungen mit über- und unterdurchschnittlichen Abschlussnoten bei unterschiedlich starker Nachfrage nach den fachspezifischen Qualifikationen sind in Abb. 3 dargestellt.

Die Ergebnisse lassen erkennen, dass Lehrabgänger mit unterdurchschnittlichen Abschlussnoten zunehmend den Beruf wechseln, wenn sie weniger passende 

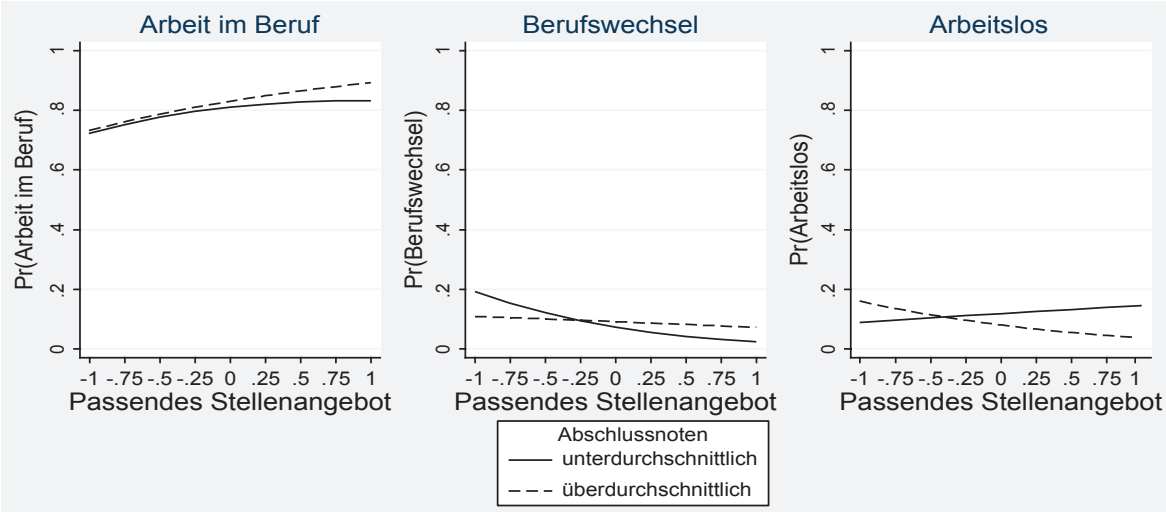

Stellenangebot ist $z$-standardisiert; $n=1358$.

Abb. 3 Geschätzte Wahrscheinlichkeit der Berufseinstiegsalternativen nach Abschussnoten und Stellenangebot.

offene Stellen vorfinden. Dazu passt auch, dass mit zunehmendem Stellenangebot das Arbeitslosigkeitsrisiko nur bei Absolventen mit guten Lehrabschlussnoten sinkt, nicht aber bei Absolventen mit schlechten Noten. Die Berufswechselneigung von Absolventen mit überdurchschnittlichen Noten ist weiter kaum von der facheigenen Qualifikationsnachfrage abhängig. Es kann davon ausgegangen werden, dass es sich hier mehrheitlich um freiwillige Wechsel handelt, wie sie durch Humankapital- oder Passungstheorie erklärt werden können. Die Resultate weisen auch darauf hin, dass Absolventen mit guten Abschlussnoten insbesondere bei einem großen Personalbedarf vom positiven Signal profitieren können. Das Ergebnis stützt somit die anhand der Signaling-Theorie getroffenen Erwartung, dass die Betriebe gute Noten als ein positives Signal für die Produktivität betrachten. Jedoch eher bei einem starken Arbeitskräftenachfrage und nicht bei einer schwachen, wie wir anhand der LabourQueue-Theorie erwartet haben (Hypothese H8). Die Resultate weisen aber darauf hin, dass Unterschiede in den Berufseinstiegschancen zwischen den Personengruppen manchmal erst sichtbar werden, wenn der Einfluss individueller Merkmale bei unterschiedlich grossem Umfang der Beschäftigungsmöglichkeiten betrachtet wird.

In den theoretischen Überlegungen zum Einfluss individueller Merkmale bei unterschiedlichem Personalbedarf haben wir weiter festgehalten, dass die Zufriedenheit mit der Ausbildung den Berufseintritt stärker beeinflussen dürfte, wenn das facheigene Stellenangebot klein ist (Abb. 4). Die Zufriedenheit mit dem Beruf ist als Ausdruck der Passung von persönlichen Charakteristiken mit der Berufswahl zu verstehen. Diese erhöht die Suchintensität nach einer der Ausbildung entsprechenden Stelle, was vor allem bei geringen Beschäftigungsmöglichkeiten die Wahrscheinlichkeit einer fachadäquaten Beschäftigung erhöhen dürfte.

Die Ergebnisse entsprechen den Erwartungen von Passungstheorie (Holland 1959) und Self-enhancement-Ansatz (Pekrun 1987) und somit auch der Hypothese H9. Bei einem sinkenden passenden Stellenangebot verringert sich für die Unzufriedenen die Wahrscheinlichkeit einer adäquaten Beschäftigung. Die Berufswechselneigung steigt spiegelbildlich. Mit dem erlernten Beruf unzufriedene Personen, 

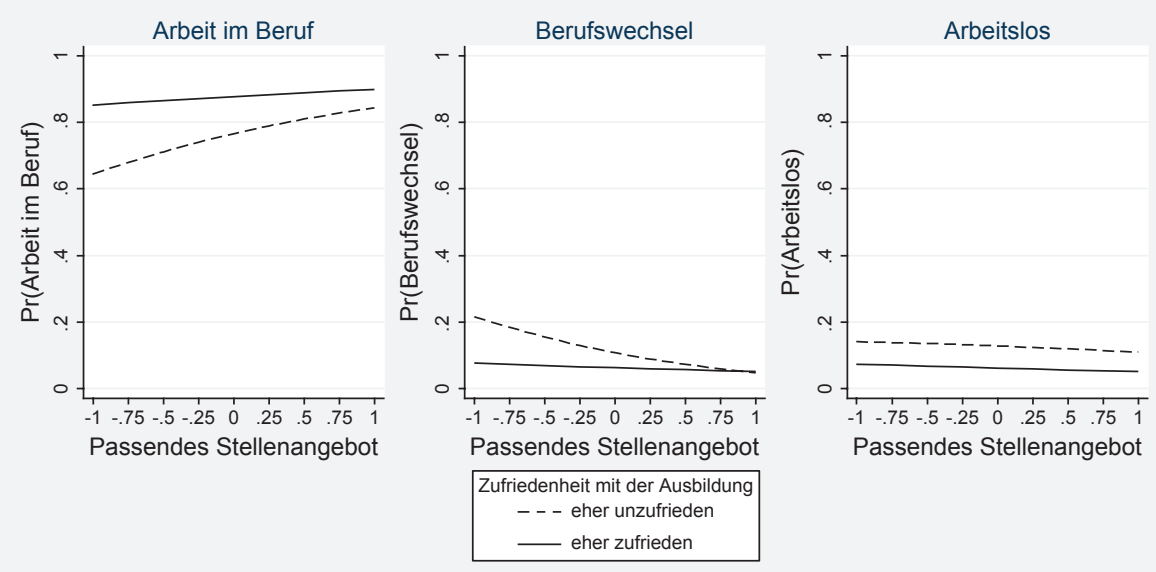

Stellenangebot ist z-standardisiert; $n=1358$.

Abb. 4 Geschätzte Wahrscheinlichkeit der Berufseinstiegsalternativen nach Zufriedenheit und Stellenangebot.

die vermutlich weniger intensiv nach einer Stelle im erlernten Beruf suchen als mit dem erlernten Beruf zufriedene Personen, werden bei schwacher fachspezifischer Qualifikationsnachfrage eher aus dem Beruf verdrängt oder suchen vermehrt nach einer fachfremden Tätigkeit. Motivationen entscheiden also vor allem bei geringen Beschäftigungsmöglichkeiten über den Berufseinstieg.

\section{Schlussfolgerungen}

In einem beruflich segmentierten Arbeitsmarkt hängt die Entwicklung des Erwerbsverlaufs wesentlich davon ab, ob die Absolventen beruflicher Grundbildungen nach Ausbildungsabschluss eine Arbeit im erlernten Beruf finden, den Beruf wechseln müssen oder arbeitslos werden. Von Interesse sind deshalb fundierte Erkenntnisse darüber, wie die auf dem Arbeitsmarkt vorgefundenen Beschäftigungsmöglichkeiten diese drei Berufseinstiegsalternativen beeinflussen. Bisher war das diesbezügliche Wissen sehr eingeschränkt, weil die Nachfrageseite des Arbeitsmarkts in der empirischen Forschung mangels geeigneter und aussagekräftiger Daten stark vernachlässigt wurde. Mit den Daten des Stellenmarktmonitors Schweiz konnten wir diese empfindliche Lücke partiell füllen, da die individuellen Beschäftigungsmöglichkeiten, welche die Berufseinsteiger antreffen, beruflich differenziert, zeitpunkt- und regionsspezifisch berücksichtigt werden konnten.

Die Analysen liefern die aufschlussreiche Erkenntnis, dass die fachliche Zusammensetzung der verfügbaren offenen Stellen eine größere Erklärungskraft für den Eintritt in einen fachlich segmentierten Arbeitsmarkt besitzt als die Gesamtarbeitsmarktlage, ausbildungsbezogene oder individuelle Merkmale. Zudem konnte gezeigt werden, dass die fachliche Zusammensetzung der offenen Stellen besonders bei einem tiefen Gesamtstellenangebot über die fachliche Adäquanz des Berufseinstiegs 
entscheidet. Es kann somit erwartet werden, dass in wirtschaftlichen Rezessionen die Zusammensetzung des Stellenangebots den Berufseinstieg noch stärker beeinflussen dürfte als hier gezeigt werden konnte.

In Verbund mit der großen Bedeutung fachspezifischer Beschäftigungsmöglichkeiten offenbart die relativ niedrige Berufswechselrate, wie ausgeprägt die fachliche Segmentierung des Arbeitsmarkts in der Schweiz ist. Neben den eingeschränkten Zugangsmöglichkeiten zu fachfremden Stellen dürften jedoch auch die in der Humankapitaltheorie postulierten Verluste berufsspezifischer Qualifikationen dazu beitragen, dass Absolventen beruflicher Grundbildungen den erlernten Beruf nicht verlassen möchten. Die verbleibenden Berufswechsel können vor allem auf eine starke Qualifikationsnachfrage in jenen Bereichen des Arbeitsmarkts zurückgeführt werden, die dem erlernten Beruf nicht entsprechen.

Das kleine Risiko eines Berufswechsels verweist weiter darauf, dass die absolvierten Berufsausbildungen und die beim Arbeitsmarkteintritt angebotenen Stellen in der Schweiz gegenwärtig fachlich gut übereinstimmen. Das berufliche Bildungssystem kann also Jugendliche nach wie vor mit jenen Qualifikationen ausstatten, die von den Betrieben auch nachgefragt werden. Sollte aber die „Akademisierung“ des Schweizer Arbeitsmarkts und eine damit einhergehende Auswahl von leistungsstarken Schüler in die allgemeine Bildung dazu führen, dass anforderungsreiche Berufsbildungen nicht mehr genügend absolviert werden, würde die fachliche Übereinstimmung von Berufsausbildungen und angebotenen Stellen abnehmen. Aufgrund der hohen Bedeutung fachspezifischer Beschäftigungsmöglichkeiten wären Probleme beim Berufseinstieg bezüglich eines Qualifikationsverlusts durch Arbeitslosigkeit oder Berufswechsel die wahrscheinliche Folge.

Der vorliegende Beitrag liefert noch eine weitere wichtige Erkenntnis, indem er empirisch nachweist, dass die gemeinsame Betrachtung nachfrage- und angebotsseitiger Wirkungskräfte den Berufseinstieg besser erklären kann als einseitige Analysen. Die Argumentation der Labour-Queue-Theorie hat sich hier als besonders fruchtbar erwiesen, als auf ihrer Basis das Zusammenspiel zwischen dem Umfang der fachspezifischen Beschäftigungsmöglichkeiten und ausbildungsbezogenen sowie individuellen Faktoren abgeleitet werden konnte. Die hier präsentierten Resultate ergänzen somit nicht nur die bestehende Forschung zum Eintritt in einen fachlich segmentierten Arbeitsmarkt. Sie verbessern auch das Verständnis über die Wirkungsweise der Angebotsseite.

Angesichts der in diesem Beitrag nachgewiesenen Bedeutsamkeit von fachlichen und fachfremden offenen Stellen für den Einstieg in den Arbeitsmarkt dürfte die zukünftige Forschung zur Entwicklung von Berufs- und Erwerbverläufen von einer stärkeren Beachtung der Nachfrage nach beruflichen Qualifikationen profitieren. So wären Erkenntnisse darüber wünschenswert, inwieweit Beschäftigungsmöglichkeiten nach dem Berufseintritt darüber bestimmen, ob die Absolventen beruflicher Grundbildungen nach einem Berufswechsel oder einer Phase von Arbeitslosigkeit wieder eine Beschäftigung im erlernten Beruf finden und somit einer Entwertung der erworbenen Qualifikationen entgehen können. 


\section{Anhang}

Tab. 3 Deskriptive Statistiken

\begin{tabular}{|c|c|c|c|c|c|c|c|c|c|}
\hline \multicolumn{10}{|c|}{ Kategoriale Variablen } \\
\hline Sprachregion & $\begin{array}{l}\text { Arbeit in } \\
\text { Beruf }\end{array}$ & $N$ & $\begin{array}{l}\text { Berufs- } \\
\text { wech- } \\
\text { sel }\end{array}$ & $N$ & Arbeitslos & $N$ & Total & $N$ & Anteile \\
\hline Deutschschweiz & 0,85 & 749 & 0,08 & 63 & 0,07 & 56 & 1,00 & 868 & 0,80 \\
\hline $\begin{array}{l}\text { Lateinische } \\
\text { Schweiz }\end{array}$ & 0,72 & 370 & 0,07 & 45 & 0,21 & 75 & 1,00 & 490 & 0,20 \\
\hline \multicolumn{10}{|l|}{ Ausbildungstyp } \\
\hline Dual ohne FHZ & 0,84 & 806 & 0,07 & 65 & 0,08 & 77 & 1,00 & 948 & 0,78 \\
\hline Dual mit FHZ & 0,86 & 225 & 0,06 & 13 & 0,08 & 19 & 1,00 & 257 & 0,15 \\
\hline $\begin{array}{l}\text { Schulisch ohne } \\
\text { FHZ }\end{array}$ & 0,42 & 46 & 0,27 & 16 & 0,31 & 26 & 1,00 & 88 & 0,04 \\
\hline Schulisch mit FHZ & 0,63 & 42 & 0,25 & 14 & 0,11 & 9 & 1,00 & 65 & 0,02 \\
\hline \multicolumn{10}{|c|}{ Ausbildungsdauer } \\
\hline 2 Jahre & 0,83 & 97 & 0,05 & 7 & 0,11 & 13 & 1,00 & 117 & 0,09 \\
\hline 3 Jahre & 0,81 & 768 & 0,10 & 88 & 0,09 & 88 & 1,00 & 944 & 0,67 \\
\hline 4 Jahre & 0,86 & 254 & 0,03 & 13 & 0,11 & 30 & 1,00 & 297 & 0,21 \\
\hline \multicolumn{10}{|c|}{ Anforderungsniveau } \\
\hline Tief & 0,83 & 624 & 0,08 & 55 & 0,09 & 59 & 1,00 & 738 & 0,66 \\
\hline Hoch & 0,80 & 495 & 0,09 & 53 & 0,11 & 72 & 1,00 & 620 & 0,34 \\
\hline \multicolumn{10}{|l|}{ Frau } \\
\hline Mann & 0,83 & 492 & 0,07 & 50 & 0,10 & 67 & 1,00 & 609 & 0,40 \\
\hline Frau & 0,82 & 627 & 0,09 & 58 & 0,08 & 64 & 1,00 & 749 & 0,60 \\
\hline \multicolumn{10}{|c|}{ Migrationshintergrund } \\
\hline Nein & 0,83 & 925 & 0,08 & 82 & 0,09 & 94 & 1,00 & 1,101 & 0,74 \\
\hline $\begin{array}{l}\text { Migrationshinter- } \\
\text { grund }\end{array}$ & 0,80 & 194 & 0,09 & 26 & 0,11 & 37 & 1,00 & 257 & 0,26 \\
\hline \multicolumn{10}{|c|}{ Metrische Variablen } \\
\hline \multicolumn{3}{|c|}{ Gesamtstellenangebot (standardisiert) } & \multicolumn{2}{|l|}{ Mean } & \multicolumn{2}{|l|}{ Std. Err. } & $\begin{array}{l}95 \% \\
\text { Conf. }\end{array}$ & \multicolumn{2}{|l|}{ Interval } \\
\hline \multicolumn{3}{|l|}{ Arbeit im Beruf } & \multicolumn{2}{|l|}{0,062} & \multicolumn{2}{|l|}{0,058} & $-0,051$ & \multicolumn{2}{|l|}{0,175} \\
\hline \multicolumn{3}{|l|}{ Berufswechsel } & \multicolumn{2}{|l|}{0,040} & \multicolumn{2}{|l|}{0,098} & $-0,153$ & \multicolumn{2}{|l|}{0,233} \\
\hline \multicolumn{3}{|l|}{ Arbeitslos } & \multicolumn{2}{|l|}{$-0,580$} & \multicolumn{2}{|l|}{0,193} & $-0,960$ & \multicolumn{2}{|l|}{$-0,199$} \\
\hline \multicolumn{3}{|c|}{ Abschlussnote Ausbildung } & \multicolumn{2}{|l|}{4,765} & \multicolumn{2}{|l|}{0,017} & 4,732 & \multicolumn{2}{|l|}{7,799} \\
\hline Arbeit im Beruf & & & 4,776 & & 0,018 & & 4,740 & 4,813 & \\
\hline Berufswechsel & & & 4,736 & & 0,067 & & 4,604 & 4,868 & \\
\hline Arbeitslos & & & 4,695 & & 0,043 & & 4,611 & 4,779 & \\
\hline $\begin{array}{l}\text { Zufriedenheit mit } \\
\text { (zentriert) }\end{array}$ & der Ausbi & lung & 0,000 & & 0,061 & & $-0,120$ & 0,120 & \\
\hline Arbeit im Beruf & & & 0,081 & & 0,082 & & $-0,080$ & 0,241 & \\
\hline Berufswechsel & & & $-0,379$ & & 0,228 & & $-0,828$ & 0,070 & \\
\hline Arbeitslos & & & $-0,315$ & & 0,157 & & $-0,624$ & $-0,006$ & \\
\hline
\end{tabular}

FHZ Fachhochschulzugang 
Tab. 4 Univariate multinomiale logistische Regression

\begin{tabular}{|c|c|c|c|c|c|}
\hline \multicolumn{4}{|l|}{ Marginaleffekte } & \multicolumn{2}{|c|}{$\begin{array}{l}\text { Koeffizienten Referenz: } \\
\text { Arbeit im Beruf }\end{array}$} \\
\hline Variable & Arbeit im Beruf & Berufswechsel & Arbeitslos & Berufswechsel & Arbeitslos \\
\hline \multicolumn{6}{|l|}{$\overline{\text { Stellenangebot }}$} \\
\hline $\begin{array}{l}\text { Im Beruf für } \\
\text { Einsteiger }\end{array}$ & $0,043 * * *$ & $-0,181^{*}$ & $-0,222 * * *$ & $-2,834 * * *$ & $-2,913 * *$ \\
\hline $\begin{array}{l}\text { In entfernten } \\
\text { Berufen }\end{array}$ & $0,013 * *$ & 0,002 & $-0,016^{* * *}$ & $-0,012$ & $-0,189 * * *$ \\
\hline Gesamt & $0,012 * * *$ & 0,001 & $-0,013 * * *$ & $-0,006$ & $-0,161 * * *$ \\
\hline $\begin{array}{l}\text { Romandie oder } \\
\text { Tessin }\end{array}$ & $-0,095 * *$ & $-0,011$ & $0,106 * * *$ & 0,028 & $1,290 * * *$ \\
\hline \multicolumn{6}{|c|}{ Ausbildungstyp (Referenz: Dual ohne FHZ) } \\
\hline Dual mit FHZ & $-0,022$ & $-0,015$ & 0,007 & $-0,262$ & $-0,109$ \\
\hline $\begin{array}{l}\text { Schulisch ohne } \\
\text { FHZ }\end{array}$ & $-0,422 * * *$ & $0,200 * * *$ & $0,222 * * *$ & $2,089 * * *$ & $1,978 * * *$ \\
\hline $\begin{array}{l}\text { Schulisch mit } \\
\text { FHZ }\end{array}$ & $-0,215^{* *}$ & $0,187 * *$ & 0,028 & $1,542 * * *$ & 0,574 \\
\hline \multicolumn{6}{|c|}{ Ausbildungsdauer (Referenz: 3 Jahre) } \\
\hline 2 Jahre & 0,025 & $-0,049$ & 0,025 & $-0,715$ & 0,220 \\
\hline 4 Jahre & 0,048 & $-0,072 * * *$ & 0,024 & $-1,338 * * *$ & 0,191 \\
\hline $\begin{array}{l}\text { Anforderungs- } \\
\text { niveau hoch }\end{array}$ & $-0,032$ & 0,015 & 0,017 & 0,226 & 0,219 \\
\hline KV & $-0,069 * *$ & 0,034 & 0,035 & $0,514^{*}$ & 0,463 \\
\hline Frau & 0,001 & 0,020 & $-0,021$ & 0,251 & $-0,226$ \\
\hline $\begin{array}{l}\text { Migrationshinter- } \\
\text { grund }\end{array}$ & 0,031 & 0,008 & 0,023 & 0,009 & 0,286 \\
\hline $\begin{array}{l}\text { Lesekompetenzen } \\
\text { PISA }\end{array}$ & $-0,055$ & $-0,077$ & 0,132 & $-0,924$ & 1,478 \\
\hline $\begin{array}{l}\text { Abschlussnote } \\
\text { Ausbildung }\end{array}$ & $-0,086$ & $-0,022$ & $-0,064^{*}$ & $-0,345$ & $-0,785^{*}$ \\
\hline $\begin{array}{l}\text { Mit Ausbildung } \\
\text { zufrieden }\end{array}$ & $0,037 * *$ & $-0,019$ & $-0,01$ & $-0,292 *$ & $-0,246^{* *}$ \\
\hline Cons & & & & $-1,14$ & $-2,69 * * *$ \\
\hline
\end{tabular}

FHZ Fachhochschulzugang

$* p<0,1 ; * * p<0,05 ; * * * p<0,01$ 


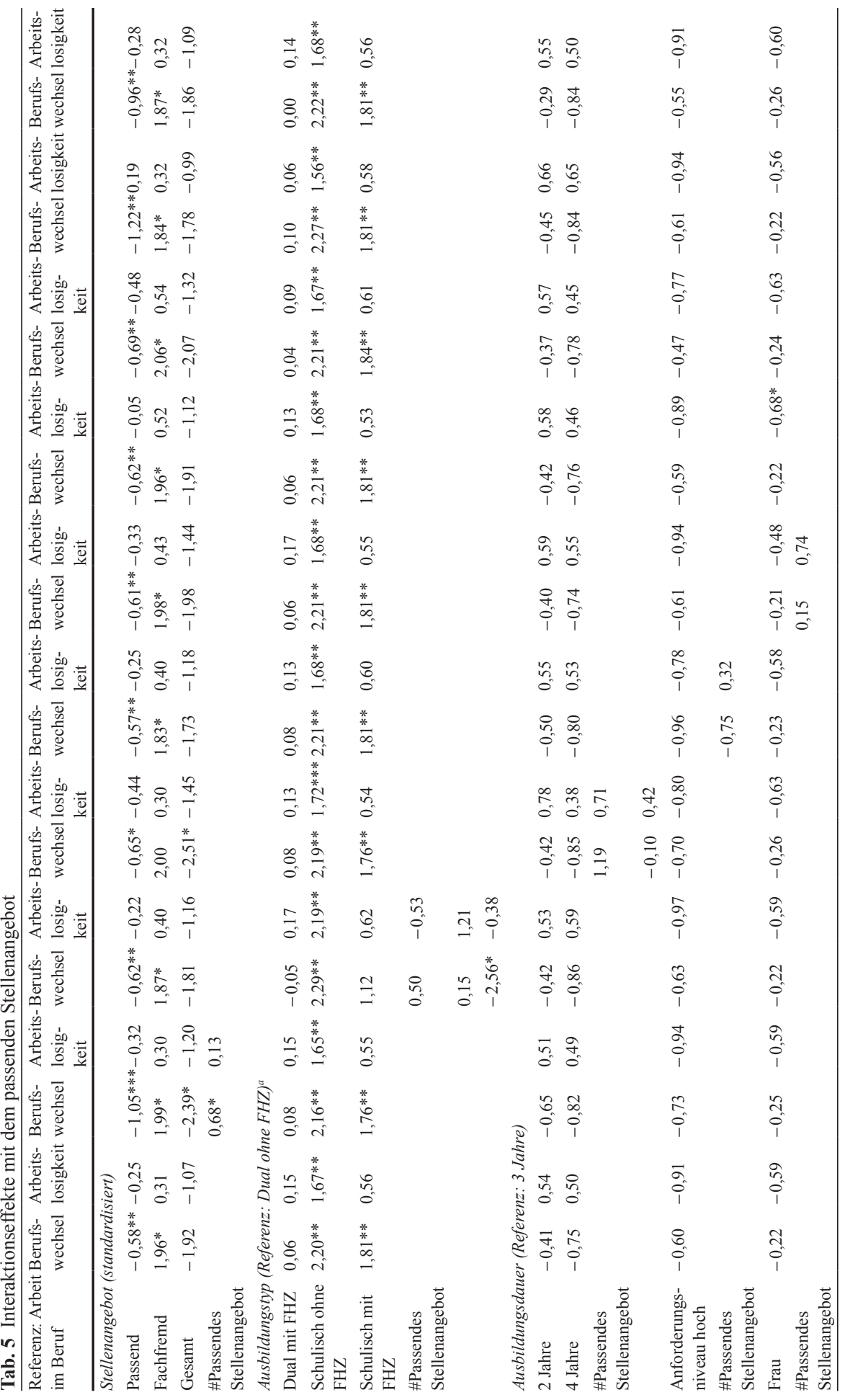




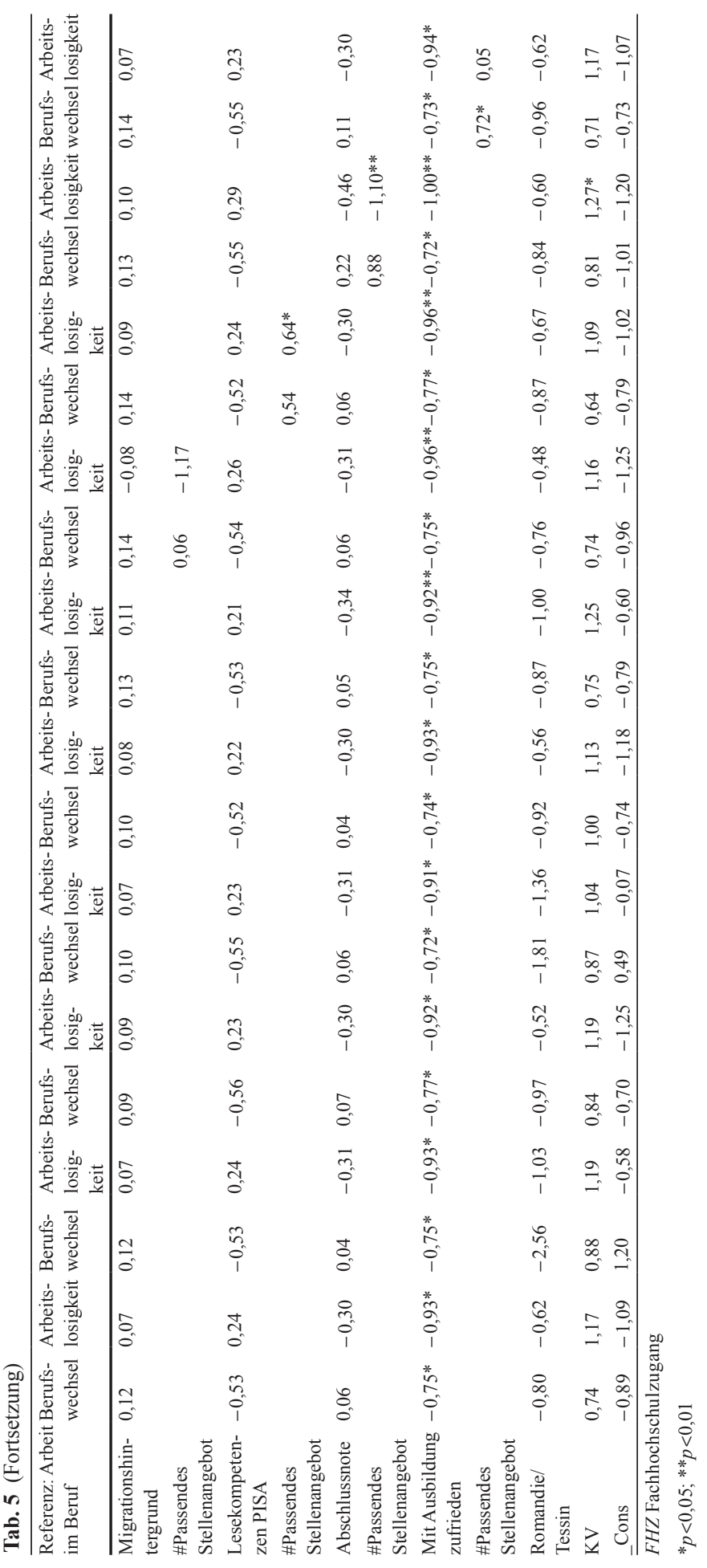




\section{Literatur}

Allmendinger, Jutta. 1989. Career mobility dynamics. A comparative analysis of the United States, Norway, and West Germany. Berlin: Max-Planck-Institut für Bildungsforschung.

Becker, Gary Stanley. 1964. Human capital. A theoretical and empirical analysis, with special reference to education. New York: National Bureau of Economic Research.

Bertschy, Kathrin, M., Cattaneo Alejandra und C. Wolter Stefan. 2008. PISA and the transition into the labour market. Labour 23:111-137.

Bielby, William T., und James N. Baron. 1986. Men and women et work: Sex segregation and statistical discrimination. American Journal of Sociology 91:759-799

Blossfeld, Hans-Peter, und Hans-Ulrich Mayer. 1988. Labor market segmentation in the Federal Republic of Germany: An empirical study of segmentation theories from a life course perspective. European Sociological Review 4:123-140.

Buchmann, Marlis, Irene Kriesi und Stefan Sacchi. 2009. Labour market, job opportunities and transitions to self-employment. European Sociological Review 25:569-583.

Büchtemann, Christoph F., Jürgen Schupp und Dana J. Soloff. 1993. Übergänge von der Schule in den Beruf. Deutschland und USA im Vergleich. Mitteilungen aus der Arbeitsmarkt- und Berufsforschung 26:507-520.

Bukodi, Erzsébet, und Shirley Dex. 2010. Bad start: Is there a way up? Gender differences in the effect of initial occupation on early career mobility in Britain. European Sociological Review 26:431-446.

Charles, Maria. 2005. Entwicklungen der beruflichen Segregation nach Geschlecht und Staatsangehörigkeit in der Schweiz 1970-2000. Demos 372005. Neuchâtel: Bundesamt für Statistik.

Charles, Maria, und Karen Bradley. 2009. Indulging our gendered selves? Sex segregation by field of study in 44 countries. American Journal of Sociology 114:924-976.

Corsten, Michael, und Wolfgang Lempert. 1997. Beruf und Moral. Weinheim: Deutscher Studien Verlag.

Dustmann, Christian, und Costas Meghir. 2005. Wages, experience and seniority. Review of Economic Studies 72:77-108.

Gangl, Markus. 2003a. Bildung und Übergangsrisiken beim Einstieg in den Beruf. Ein europäischer Vergleich zum Arbeitsmarktwert von Bildungsabschlüssen. Zeitschrift für Erziehungswissenschaft 6:72-89.

Gangl, Markus. 2003b. The only way is up? Employment protection and job mobility among recent entrants to European labour markets. European Sociological Review 19:429-449.

Gangl, Markus. 2004. Welfare states and the scar effects of unemployment: A comparative analysis of the United States and West Germany. American Journal of Sociology 109:1319-1364.

Gerber-Schenk, Michelle, Benno Rottermann und Markus P. Neuenschwander. 2010. Passungswahrnehmung, Selbstkonzept und Jugendarbeitslosigkeit. In Schulübergang und Selektion, Hrsg. Hans-Ulrich Grunder und Markus P. Neuenschwander, 121-130. Chur: Rüegger.

Helbling, Laura, und Stefan Sacchi. 2014. Scarring effects of early unemployment among young workers with vocational credentials in Switzerland. Empirical Research in Vocational Education and Training 6:12.

Holland, John L. 1959. A theory of vocational choice. Journal of Counseling Psychology 6:35-45.

Konietzka, Dirk. 2010. Berufliche Ausbildung und der Übergang in den Arbeitsmarkt. In Bildung als Privileg? Erklärungen und Befunde zu den Ursachen der Bildungsungleichheit, Hrsg. Rolf Becker und Wolfgang Lauterbach, 277-304. Wiesbaden: VS Verlag für Sozialwissenschaften.

Konietzka, Dirk. 2011. Berufsbildung im sozialen Wandel. In Lehrbuch der Bildungssoziologie, Hrsg. Rolf Becker, 265-288. Wiesbaden: VS Verlag für Sozialwissenschaften.

Kriesi, Irene. 2006. Beschäftigungsmöglichkeiten und Erwerbskontinuität von Frauen: Zur Bedeutung des Stellenangebots für Erwerbsunterbrechungen und Wiedereintritte ins Berufsleben. Zürich: Rüegger.

Kriesi, Irene, Marlis Buchmann und Stefan Sacchi. 2010. Variation in job opportunities for men and women in the Swiss labour market 1962-1989. Research in Social Stratification and Mobility 28:309-323.

Long, J. Scott, und Freese Jeremy. 2006. Regression models for categorical dependent variables using Stata. 2. Aufl. College Station: Stata Press Publications.

Margolis, David, Eric Plug, Véronique Simonnet und Lars Vilhuber. 2004. Early career experiences and later career outcomes: An international comparison. In Human capital over the life cycle - A European perspective, Hrsg. Catherine Sofer. London: Edward Elgar.

Mincer, Jacob. 1974. Schooling, experience and earnings. New York: National Bureau of Economic Research. 
Mortensen, Dale T. 1986. Job search and labour market analysis. Handbook of Labour Economics 15:849-919.

Müller, Barbara, und Jürg Schweri. 2009. Berufswechsel beim Übergang von der Lehre in den Arbeitsmarkt. Schweizerische Zeitschrift für Bildungswissenschaften 31:199-227.

Müller, Barbara, und Jürg Schweri. 2015. How specific is apprenticeship training? Evidence from interfirm and occupational mobility after graduation. Oxford economic Papers. First published online: doi: 10.1093/oep/gpv040.

Müller, Barbara, und Stefan C. Wolter. 2013. The role of hard-to obtain information on ability for the school-to-work transition. Empirical Economics. Published online first: 14. May 2013. http://dx.doi. org/10.1007/s00181-013-0709-2.

Pekrun, Reinhard. 1987. Die Entwicklung leistungsbezogener Identität bei Schülern. In Identität: Entwicklungen psychologischer und soziologischer Forschung, Hrsg. Hans-Peter Frey, und Karin Hausser, 43-57. Stuttgart: Enke.

Pollmann-Schult, Matthias, und Felix Büchel. 2002. Ausbildungsinadäquate Erwerbstätigkeit: eine berufliche Sackgasse? Eine Analyse für jüngere Nicht-Akademiker in Westdeutschland. Mitteilungen aus der Arbeitsmarkt- und Berufsforschung 35:371-384.

Reskin, Barbara, und Patricia A. Roos. 1990. Job queues, gender queues. Explaining women's inroads into male occupations. Philadelphia: Temple University Press.

Rogerson, Richard, Robert Shimer und Randall Wright. 2005. Search-theoretic models of the labour market: A survey. Journal of Economic Literature 43:959-988.

Sacchi, Stefan. 2008. TREE-Längsschnittgewichtung: Konstruktion und Anwendung. Dokumentation zu den acht Erhebungswellen 2000 bis 2007. Bern: TREE und cue Sozialforschung.

Sacchi, Stefan, und Alexander Salvisberg. 2012. Berufseinstiegs-Barometer 2012. Report im Auftrag des Bundesamts für Berufsbildung und Technologie (BBT). Bern: Bundesamt für Berufsbildung und Technologie.

Sacchi, Stefan, und Alexander Salvisberg. 2014. Arbeitsmarktperspektiven von Fachkräften aus unterschiedlichen Berufen 2013. Report im Auftrag des Staatssekretariats für Bildung, Forschung und Innovation SBFI. Zürich: Universität Zürich, Stellenmarkt-Monitor Schweiz.

Sacchi, Stefan, Alexander Salvisberg und Marlis Buchmann. 2005. Long-term dynamics of skill demand in Switzerland, 1950-2000. In Contemporary Switzerland. Revisiting the special case, Hrsg. Hanspeter Kriesi, Peter Farago, Martin Kohli und Milad Zarin-Nejadan, 105-134. Basingstoke: Palgrave Macmillan.

Salvisberg, Alexander, und Stefan Sacchi. 2014. Labor market prospects of Swiss career entrants after completion of VET. European Societies 16:255-274.

Schellenbauer, Patrik, Rudolf Walser, Daniela Lepori, Beat Hotz-Hart und Philipp Gonon. 2010. Die Zukunft der Lehre. Die Berufsbildung in einer neuen Wirklichkeit. Zürich: Avenir Suisse.

Scherer, Stefanie. 2004. Stepping-stones or traps? The consequences of labour market entry positions on future careers in West Germany, Great Britain and Italy. Work, Employment and Society 18:369-394.

Sengenberger, Werner. 1978. Arbeitsmarktstruktur. Ansätze zu einem Modell des segmentierten Arbeitsmarkts. 2. Aufl. Frankfurt a. M: Campus.

Sheldon, George. 2005. Der berufsstrukturelle Wandel der Beschäftigung in der Schweiz 1970-2000. Ausmass, Ursachen und Folgen. Neuchâtel: Bundesamt für Statistik.

Solga, Heike, und Dirk Konietzka. 1999. Occupational matching and social stratification. Theoretical Insights and empirical observations Observations Taken from a German-German Comparison. European Sociological Review 15:25-47.

Spence, Michael. 1973. Job market signaling. The Quarterly Journal of Economics 87:355-374.

Stalder, Barbara E. 2011. Das intellektuelle Anforderungsniveau beruflicher Grundbildungen in der Schweiz. Ratings der Jahre 1999-2005. Basel: TREE/Institut für Soziologie der Universität Basel.

Stalder, Barbara E., und Christoph Nägele. 2011. Vocational education and training in Switzerland: Organisation, development and challenges for the future. In Transitionen im Jugendalter. Ergebnisse der Schweizer Längsschnittstudie, Hrsg. Max Bergman, Sandra Hupka-Brunner, Anita Keller, Thomas Meyer und Barbara E. Stalder, 18-39. Zürich: Seismo.

Thurow, Lester C. 1975. Generating inequality. Mechanisms of distribution in the U.S. economy. New York: University of California, Basic Books.

Thurow, Lester C. 1978. Die Arbeitskräfteschlange und das Modell des Arbeitsplatzwettbewerbs. In Der gespaltene Arbeitsmarkt. Probleme der Arbeitsmarktsegmentation, Hrsg. Werner Sengenberger, 117-138. Frankfurt a. M: Campus.

TREE. Hrsg. 2011. Projektdokumentation 2000-2007. Basel: TREE. 
Vandenberghe, Vincent. 2010. The long-term effects of recessions on youth. Reviewing the evidence. Université catholique de Louvain (UCL): Louvain-la-Neuve.

Williams, Richard. 2012. Using the margins command to estimate and interpret adjusted predictions and marginal effects. The Stata Journal 12:308-331.

Witte, James C., und Arne L. Kalleberg. 1995. Matching training and jobs: The fit between vocational education and employment in the German Labour Market. European Sociological Review 11:293-317.

Wolter, Stefan C., und Paul Ryan. 2011. Apprenticeship. In Handbook of economics of education, Hrsg. Eric A. Hanushek, Stephen Machin und Ludger Wössmann, 521-576. Amsterdam: Elsevier.

Helen Buchs, 1983, lic. phil., wissenschaftliche Assistentin, Institut für Soziologie, Universität Zürich. Forschungsgebiete: Bildung Arbeitsmarkt, Beruf, Personalsuche. Veröffentlichungen: Personalsuche und Stellenbesetzung. Empirische Analysen zur Praxis der Personalsuche, betrieblichen Rekrutierungsproblemen und zur Personalsuche im Ausland. Zürich 2012 (mit S.Sacchi).

Barbara Müller, 1976, lic. rer. oec., wissenschaftliche Mitarbeiterin, Institut für Soziologie, Projekt TREE, Universität Bern. Forschungsgebiete: Bildung und Arbeitsmarkt. Veröffentlichungen: How specific is apprenticeship training? Evidence from inter-firm and occupational mobility after graduation, Oxford Economic Papers doi: 10.1093/oep/gpv040, 2015 (mit J.Schweri); Leistung oder soziale Herkunft? Wiesbaden 2015 (mit S. Hupka-Brunner, K. Scharenberg und T. Meyer); The role of hard-to-obtain information on ability for the school-to-work transition. Empirical Economics 46, 2014 (mit S.C. Wolter); Berufswechsel beim Übergang von der Lehre in den Arbeitsmarkt. Schweizerische Zeitschrift für Bildungswissenschaften 31, 2009 (mit J.Schweri).

Marlis Buchmann, 1950, Dr. phil. Professorin für Soziologie an der Universität Zürich und Direktorin des Jacobs Center for Productive Youth Development Universität Zürich. Forschungsgebiete: Soziologie des Lebenslaufs, soziale Ungleichheit, Bildung, Beruf und Arbeitsmarkt, kultureller Wandel. Veröffentlichungen: Beginning school transition and academic achievement in mid-elementary school: Does gender matter? Gender differences in aspirations and attainment: A life course perspective. Cambridge 2014; Geschlechtstypische Berufswahl: Begabungszuschreibungen, Aspirationen und Institutionen. Sonderheft 52 der Kölner Zeitschrift für Soziologie und Sozialpsychologie 2012; The future of young women's economic role in a globalized economy: New opportunities - persisting constraints. New Directions in Youth Development 135, 2012 (mit T. Malti); Transition to adulthood in Europe. Annual Review of Sociology 37, 2011 (mit I. Kriesi). 